\title{
Dinámica de la estructura de la comunidad bentónica en las formaciones coralinas someras del archipiélago de San Bernardo, Caribe colombiano
}

\section{Dynamics of the benthic community structure on the shallow coral formations of the San Bernardo Archipelago, Colombian Caribbean}

\author{
Johanna Vega-Sequeda ${ }^{I^{*}}$, Claudia Agudelo-Ramírez ${ }^{2}$, Álvaro Mendoza-Mazzeo ${ }^{3}$ y Adolfo Sanjuan-Muñoz ${ }^{4}$ \\ $\begin{array}{ll}\text { (D) } 0000-0003-2507-8560 & \text { (D) } 0000-0001-9800-6673\end{array}$ \\ (D) $0000-0002-3539-528 X$ \\ (iD) $0000-0002-4786-862 X$
}

1. Investigador independiente. johavega@gmail.com*

2. Sanjuan y Asociados Ltda. clauctg@hotmail.com

3. Hyser Ltda., Barranquilla, Colombia.Imendozamazzeo@gmail.com

4. Departamento de Ciencias Biológicas y Ambientales, Facultad de Ciencias Naturales e Ingeniería, Universidad Jorge Tadeo Lozano, Sede Santa Marta, El Rodadero, Santa Marta, Colombia.adolfo.sanjuan@utadeo.edu.co

* Autora de correspondencia.

\section{RESUMEN}

$\mathrm{L}$

os arrecifes de coral han sufrido una extensa degradación en las últimas décadas como resultado de perturbaciones antropogénicas y naturales. Este estudio presenta una caracterización histórica (1989-2015) de los cambios en la composición del sustrato en formaciones coralinas en el archipiélago de San Bernardo. Adicionalmente, en el 2015 se evaluó la salud arrecifal (presencia de signos de deterioro) y se analizaron datos satelitales de temperatura. En general, la cobertura de coral mostró una tendencia estable entre 1989 y 2010. En 2013 y 2015 se registraron diferencias en el ensamblaje coralino las cuales pueden estar asociadas al incremento en la cobertura de las especies de los géneros Orbicella, Porites, Agaricia, Siderastrea y del hidrocoral Millepora complanata. Las menores coberturas observadas en 1989, 1991 y 2005 estuvieron posiblemente relacionados con eventos de mortalidades masivas de especies arrecifales por enfermedades y blanqueamiento. Se registró un comportamiento opuesto entre el sustrato abiótico y las algas, siendo este último grupo el componente dominante del sustrato desde el 2013. Espacialmente se presentaron diferentes tendencias. Las estaciones en Ceycen, Tintipán y Mangle presentaron mejores condiciones de desarrollo coralino en el tiempo, mientras que las estaciones en Cabruna y Palma, lugares más cercanos a los centros urbanos, presentaron indicios de tendencia hacia el deterioro. Adicionalmente, a partir de junio del 2015 se registraron valores de temperatura por encima del umbral de tolerancia al blanqueamiento $\left(29,4^{\circ} \mathrm{C}\right)$, por lo que posiblemente los corales enfrentaron un estrés térmico que causó blanqueamiento en 18 de las 19 estaciones evaluadas. Son esenciales las acciones de gestión sobre los impactos locales, acompañados de la información suministrada por los programas de monitoreo, para que los arrecifes puedan recuperarse de los impactos naturales y antropogénicos que los afectan.

PALABRAS CLAVE: arrecifes coralinos, monitoreo coralino, cobertura arrecifal, Parque Nacional Natural Corales del Rosario y San Bernardo, Caribe colombiano

\section{ABSTRACT}

$\mathrm{C}$ oral reefs have experienced an extensive degradation over the last decades as a result of anthropogenic and natural disturbances. This study presents an historical characterization (1989-2015) of changes in composition of the substrate in coral formations in the San Bernardo Archipelago. In cover showed a stable trend between 1989 and 2010. The observed differences in the coral reef formations in 2013 and 2015 could have been due to the increase in coverage of species of the genus Orbicella, Porites, Agaricia, Siderastrea and the hydrocoral Millepora complanata. The lower coverage in 1989, 1991 and 2005 was possibly related to massive mortality events of reef species due to diseases and bleaching. Abiotic substrate and algal cover exhibited opposing behavior, and algae became the dominant component of the substrate since 2013. Spatially, there were different trends: stations at Ceycen, Tintipán and Mangle presented better coral development conditions over time, while stations at Cabruna and Palma, closer to urban centers, showed signs of deterioration. In addition, temperatures above the bleaching tolerance threshold $\left(29.4^{\circ} \mathrm{C}\right)$ were recorded since June 2015 , which is possibly why corals faced thermal stress and bleached in 18 of the 19 evaluated stations. Management actions on local impacts are essential, accompanied by information provided by monitoring programs, so that coral reefs can recover from the natural and anthropogenic impacts that affect them.

KEY WORDS: coral reefs, coral monitoring, reef cover, Corales del Rosario y San Bernardo Natural National Park, Colombian Caribbean 


\section{INTRODUCCIÓN}

Los arrecifes de coral constituyen uno de los ecosistemas más valiosos, productivos y biodiversos del planeta, ofreciendo variados servicios ecosistémicos a la población costera; son fuente de gran valor económico para la pesca tradicional y el turismo (Birkeland, 1997; Buddemeier et al., 2004; Burke et al., 2011). Este ecosistema es muy sensible a cambios en su entorno, por lo que ha sido catalogado como un indicador de perturbaciones ambientales (Zea, 1991; Bernhard, 2007). Por ello, la variación temporal de la cobertura coralina es considerada un buen indicador del estado de salud de los arrecifes coralinos, debido a que los corales son los constructores fundamentales del ecosistema (Birkeland, 1997; Hughes et al., 2010).

A pesar de su valor, los arrecifes coralinos han sufrido una extensa degradación en las últimas décadas y cerca del $75 \%$ de ellos están bajo amenaza como resultado de perturbaciones antropogénicas y naturales (Burke et al., 2011). Factores como la sobrepesca, la pesca destructiva, la contaminación, las altas tasas de sedimentación, las enfermedades, el blanqueamiento y el cambio climático, son las causas más importantes de deterioro identificadas a nivel mundial y nacional, incluyendo el área de las islas de San Bernardo, en el Caribe colombiano (Birkeland, 1997; Díaz et al., 2000; Wilkinson y Souter, 2008; Burke et al., 2011).

Si bien se han realizado algunos estudios en los arrecifes coralinos del archipiélago de San Bernardo desde 1975 (Erhardt y Meinel, 1975; Duque y Gómez, 1983; Prahl y Erhardt, 1985; Díaz et al., 2000; López-Victoria y Díaz, 2000; Garzón-Ferreira y Díaz, 2003), son escasas las investigaciones que han evaluado la dinámica de los arrecifes del área en una amplia escala espacial y temporal. Se destaca la revisión de Alvarado et al. (2011) y la información suministrada por el Sistema Nacional de Monitoreo de Arrecifes Coralinos (SIMAC) que en San Bernardo inició actividades desde 2002 (Rodríguez-Ramírez et al., 2010; Navas-Camacho et al., 2011; Bastidas et al., 2014).

Desde la perspectiva del manejo y ante la problemática del cambio climático, es importante entender la dinámica de los ecosistemas en el tiempo y detectar sus cambios (Vega-Sequeda et al., 2017). En las formaciones coralinas del archipiélago de San Bernardo se realizó un monitoreo de cinco muestreos entre 1989 y 2010, en el marco del programa de Seguimiento y Monitoreo de Ecosistemas Naturales Marinos del Plan de Manejo Ambiental del Terminal de Coveñas de Ecopetrol S.A. (la empresa petrolera

\section{INTRODUCTION}

Coral reefs constitute one of the most valuable, productive, and biodiverse ecosystems on the planet, offering various ecosystem services to the coastal population; they are a source of great economic value for traditional fishing and tourism (Birkeland, 1997; Buddemeier et al., 2004; Burke et al., 2011). This ecosystem is very sensitive to changes in its environment, which is why it has been classified as an indicator of environmental disturbances (Zea, 1991; Bernhard, 2007). For this reason, the temporal variation of the coral cover is considered a good indicator of the health status of coral reefs, since corals are the fundamental constructors of the ecosystem (Birkeland, 1997; Hughes et al., 2010).

Despite their value, coral reefs have suffered extensive degradation in recent decades and about $75 \%$ of them are under threat as a result of anthropogenic and natural disturbances (Burke et al., 2011). Factors such as overfishing, destructive fishing, pollution, high rates of sedimentation, disease, bleaching, and climate change, are the most important causes of deterioration identified globally and nationally, including the San Bernardo's islands area, in the Colombian Caribbean (Birkeland, 1997; Díaz et al., 2000; Wilkinson and Souter, 2008; Burke et al., 2011).

Although some studies have been carried out in the coral reefs of the San Bernardo archipelago since 1975 (Erhardt and Meinel, 1975; Duque and Gómez, 1983; Prahl and Erhardt, 1985; Díaz et al., 2000; López-Victoria and Díaz, 2000; Garzón-Ferreira and Díaz, 2003), there are few investigations that have evaluated the dynamics of the reefs in the area on a wide spatial and temporal scale. It is highlight the review by Alvarado et al (2011), as well as, the information provided by the National Coral Reef Monitoring System (SIMAC), which in San Bernardo began activities since 2002 (Rodríguez-Ramírez et al., 2010; Navas-Camacho et al., 2011; Bastidas et al., 2014).

From the perspective of management and faced with the problem of climate change, it is important to understand the dynamics of ecosystems over time and detect their changes (Vega-Sequeda et al., 2017). In the coral formations of the San Bernardo archipelago, monitoring of five samplings was carried out between 1989 and 2010, within the framework of the Monitoring and Monitoring Program for Marine Natural Ecosystems of the Environmental Management Plan of the Coveñas 
estatal colombiana), en el que se evaluó la cobertura del sustrato y la composición, abundancia y diversidad coralina. Adicionalmente, se llevaron a cabo campañas de muestreo adicionales en 2013 y 2015. En esta investigación se analizaron los cambios temporales en la cobertura y composición de las formaciones coralinas, así como el ensamblaje de corales escleractinios en el archipiélago de San Bernardo entre 1989 у 2015.

\section{ÁREA DE ESTUDIO}

El archipiélago de San Bernardo está localizado frente a la costa del departamento de Sucre en el Caribe colombiano (Figura 1). Este archipiélago está compuesto por 12 islas y 4 islotes, destacándose el islote artificial de Santa Cruz del Islote como el que tiene la mayor población humana nativa, la cual deriva su sustento principalmente de la pesca artesanal y en menor medida del turismo (Duque-Rico y Torres-Gómez, 2011). El área sumergida hace parte del Parque Nacional Natural Corales del Rosario y San Bernardo (PNNCRSB), que abarca un área marina protegida de 120000 ha (Zarza-González, 2011).
Terminal of Ecopetrol S.A. (the oil company Colombian state), in which the substrate coverage and the composition, abundance and coral diversity were evaluated. Additionally, sampling campaigns were carried out in 2013 and 2015. This research analyzed the temporal changes in the coverage and composition of the coral formations, as well as the assembly of scleractinian corals in the San Bernardo archipelago between 1989 and 2015.

\section{STUDY AREA}

The San Bernardo archipelago is located off the coast of the department of Sucre in the Colombian Caribbean (Figure 1). This archipelago is made up of 12 islands and 4 islets, highlighting the artificial islet of Santa Cruz del Islote as the one with the largest native human population, which derives its livelihood mainly from artisanal fishing and to a lesser extent from tourism (Duque-Rico and Torres-Gómez, 2011). The submerged area is part of the Corales del Rosario and San Bernardo National Natural Park (PNNCRSB) which covers a protected marine area of 120000 ha (ZarzaGonzález, 2011).

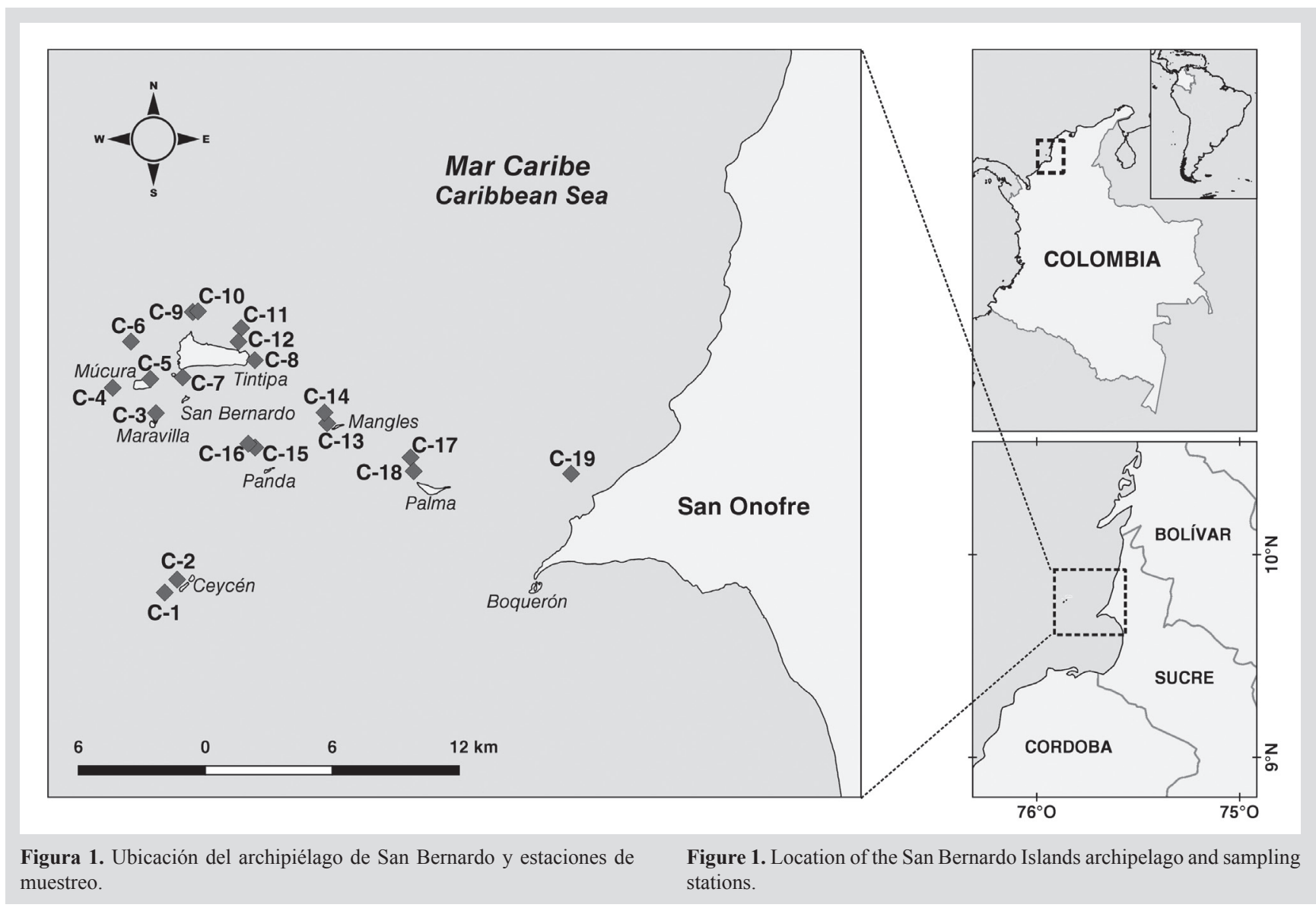


El clima de la región es tropical, con una época seca (diciembre-abril) con mayor influencia de los vientos alisios del NE, un ligero descenso de la temperatura del agua $\left(27^{\circ} \mathrm{C}\right)$ y un incremento en la acción del oleaje, así como una época de lluvias (mayo-noviembre), cuando los vientos alisios se debilitan, el agua es más cálida $\left(29^{\circ} \mathrm{C}\right)$, las lluvias son frecuentes y llegan las descargas del río Sinú que incrementan la turbidez (Pujos et al., 1986; Patiño y Flores, 1993; AndradeAmaya, 2001; Gutiérrez-Moreno et al., 2011).

El archipiélago de San Bernardo posee un complejo arrecifal parcialmente emergido con parches, arrecifes franjeantes y bancos coralinos que ocupan una extensión aproximada de 213,3 $\mathrm{km}^{2}$, de los cuales $134,5 \mathrm{~km}^{2}$ corresponden a cobertura viva, presentando un mayor desarrollo en los flancos norte y occidente. Las formaciones más destacadas de este sector se encuentran al norte y noroccidente de isla Mangle, al norte de isla Tintipán y en los costados norte y occidente de los bajos Las Nubes, Minalta y Julián (Díaz et al., 2000).

Los arrecifes coralinos de las islas de San Bernardo han sufrido fuertes impactos antropogénicos desde la década de 1980. Estos se han atribuido a enfermedades epizoóticas coralinas, sedimentación y turbidez provenientes del río Sinú (López-Victoria y Díaz, 2000; Alvarado et al., 2011), pesca con dinamita y sobrepesca, desarrollo costero, turismo incontrolado, construcción en las islas y extracción de corales para artesanías (Ramírez, 1990; Díaz et al., 2000; GarzónFerreira y Díaz, 2003; Alvarado et al., 2011).

\section{MATERIALES Y MÉTODOS}

\section{Fase de campo}

Se utilizaron los datos de los monitoreo realizados en 1989, 1991, 1999, 2005 y 2010, documentados por Ramírez (1990, 1992, 2000, 2010) e Invemar (2005). El monitoreo inicial evaluó 11 estaciones, en 1999 se ampliaron a 18 y en 2005 se añadió una más para completar 19 estaciones (Figura 1) que se encuentran entre 4 y $12 \mathrm{~m}$ de profundidad. Para cada monitoreo se siguieron las coordenadas geográficas históricas de cada punto. Las estaciones están dispuestas en los alrededores de las islas Ceycen (C-1 y C-2), Maravilla (C-3), Múcura (C-4 a C-6), Tintipán (C-7 a C-12), Mangle (C-13 y C-14), Panda (C-15 y C-16), Palma (C-17 y C-18) y Cabruna (C-19). En cada estación se estimó la cobertura del sustrato por algas, corales (por especie), otros invertebrados (poliquetos sésiles, esponjas y anémonas) y sustrato abiótico, por el método de punto intercepto en transecto. En cada sitio
The climate of the region is tropical, with a dry season (December-April) with greater influence from the NE trade winds, a slight decrease in water temperature (27 ${ }^{\circ} \mathrm{C}$ ) and an increase in the action of the waves; as well as a rainy season (May-November), when the trade winds weaken, the water is warmer $\left(29^{\circ} \mathrm{C}\right)$, the rains are frequent, and the discharges from the Sinú River that increase turbidity (Pujos et al., 1986; Patiño and Flores, 1993; Andrade-Amaya, 2001; Gutiérrez-Moreno et al., 2011).

The San Bernardo archipelago has a partially emerged reef complex with patches, fringing reefs, and coral banks that occupy an area of approximately 213.3 $\mathrm{km}^{2}$, of which $134.5 \mathrm{~km}^{2}$ corresponds to living cover, presenting greater development on the northern flanks and the west. The most prominent formations in this sector are found to the north and northwest of Mangle Island, to the north of Tintipán Island, and on the north and west sides of the Las Nubes, Minalta, and Julián lowlands (Díaz et al., 2000).

The coral reefs of the San Bernardo Islands have suffered strong anthropogenic impacts since the 1980s. These have been attributed to epizootic coral diseases, sedimentation and turbidity from the Sinú River (LópezVictoria and Díaz, 2000; Alvarado et al., 2011), dynamite fishing and overfishing, coastal development, uncontrolled tourism, construction on islands, and extraction of corals for handicrafts (Ramírez, 1990; Díaz et al., 2000; GarzónFerreira and Díaz, 2003; Alvarado et al., 2011).

\section{MATERIALS AND METHODS}

\section{Field phase}

The data from the monitoring carried out in 1989, 1991, 1999, 2005, and 2010, documented by Ramírez $(1990,1992,2000,2010)$ and Invemar (2005) were used. Initial monitoring evaluated 11 stations, in 1999 they were expanded to 18 , and in 2005 one more was added to complete 19 stations (Figure 1) that are between 4 and $12 \mathrm{~m}$ deep. For each monitoring, the historical geographic coordinates of each point were followed. The stations are arranged around the islands Ceycen ( $\mathrm{C}-1$ and $\mathrm{C}-2)$, Maravilla (C-3), Múcura (C-4 to C-6), Tintipán (C-7 to $\mathrm{C}-12$ ), Mangle (C-13 and $\mathrm{C}-14)$, Panda (C-15 and C-16), Palma (C-17 and C-18), and Cabruna (C-19). At each station, the substrate coverage by algae, corals (by species), other invertebrates (sessile polychaetes, sponges, and anemones), and abiotic substrate were estimated by 
se extendieron dos transectos no fijos de $75 \mathrm{~m}$, anotando cada $10 \mathrm{~cm}$ el componente del sustrato que estaba debajo hasta completar 1500 puntos.

En 2013 y 2015, se emplearon las categorías del sustrato descritas por Garzón-Ferreira et al. (2002) con algunas modificaciones: algas, corales duros (por especie), esponjas, corales blandos (gorgonáceos y zoantídeos), otros invertebrados y sustrato abiótico. Adicionalmente, en el 2015 se registró la presencia de enfermedades coralinas y blanqueamiento con base en Sutherland et al. (2004) y Raymundo et al. (2008).

Se obtuvieron datos de la temperatura superficial del mar y del índice "grados semanas de calentamiento" (Degree Heating Weeks - DHW) del 2015 a partir de las bases de datos satelitales de la NOAA Coral Reef Watch (2020). La serie del índice DHW muestra la cantidad de estrés térmico acumulado en los corales durante las últimas 12 semanas. Este índice considera el "umbral de tolerancia" al blanqueamiento coralino, que para el Caribe colombiano es de $29,4{ }^{\circ} \mathrm{C}$. Un DHW superior a $4{ }^{\circ} \mathrm{C}$-semanas ha demostrado un blanqueamiento significativo y valores por encima de $8{ }^{\circ} \mathrm{C}$-semanas han causado blanqueamiento masivo y mortalidad asociada (Liu et al., 2018).

\section{Análisis de la información}

Para describir el ensamblaje coralino se calcularon las medidas de riqueza específica (S), diversidad de ShannonWienner (H') y la uniformidad de Pielou (J'), calculando el promedio, error estándar y coeficiente de variación por estación. Es necesario tener en cuenta que en los resultados de los monitoreos previos al 2013 Agaricia sp. agrupó a $A$. agaricites y $A$. tenuifolia, Orbicella annularis integró a $O$. faveolata y O. Franksi, y Siderastrea sp. incluyó a S. siderea y $S$. radians.

Los patrones de variación temporal (entre años) del ensamblaje coralino fueron representados por promedios Bootstrap en gráficos de Escalamiento Multidimensional o MDS (por sus siglas en inglés) multivariada y sus respectivos intervalos Boostrap de $95 \%$, con 100 repeticiones por grupo y un coeficiente de correlación mínimo de 0,99 (Clarke y Gorley, 2015). Para determinar posibles diferencias significativas de la estructura entre años se aplicó un PERMANOVA con 999 permutaciones (Anderson, 2001). Se definieron los corales que mayor contribución tuvieron a la disimilitud entre grupos mediante el análisis SIMPER (Clarke, 1993). Al realizar las comparaciones en el ámbito local y nacional, se obviaron las diferencias derivadas de los métodos de evaluación en campo. the intercept point method in the transect. At each site, two non-fixed transects of $75 \mathrm{~m}$ were extended, scoring every $10 \mathrm{~cm}$ the component of the substrate that was underneath until completing 1500 points.

In 2013 and 2015, the substrate categories described by Garzón-Ferreira et al. (2002) with some modifications: algae, hard corals (by species), sponges, soft corals (gorgonians and zoanthids), other invertebrates, and abiotic substrate. Additionally, in 2015 the presence of coral diseases and bleaching was recorded based on Sutherland et al. (2004) and Raymundo et al. (2008).

Data for the sea surface temperature and the index "degrees of warming weeks" (Degree Heating Weeks - DHW) for 2015 were obtained from the NOAA Coral Reef Watch (2020) satellite databases. The DHW index series shows the amount of heat stress accumulated in corals during the last 12 weeks. This index considers the "tolerance threshold" for coral bleaching, which for the Colombia Caribbean is $29.4{ }^{\circ} \mathrm{C}$. A DHW greater than $4{ }^{\circ} \mathrm{C}$-weeks has shown significant whitening and values above $8{ }^{\circ} \mathrm{C}$-weeks have caused massive bleaching and associated mortality (Liu et al., 2018).

Analysis of the information

To describe the coral assemblage, the measures of specific richness (S), Shannon-Wienner diversity (H'), and Pielou uniformity (J') were calculated, calculating the average, standard error, and coefficient of variation per station. It is necessary to take into account that in the results of the monitoring before 2013 Agaricia sp. grouped A. agaricites and A. tenuifolia, Orbicella annularis integrated $O$. faveolata and O. Franksi, and Siderastrea sp. included S. siderea and $S$. radians.

The temporal variation patterns (between years) of the coral assemblage were represented by Bootstrap averages in Multidimensional Scaling graphs or multivariate MDS and their respective Boostrap intervals of $95 \%$, with 100 repetitions per group and a coefficient of Minimum correlation of 0.99 (Clarke and Gorley, 2015). To determine possible significant differences in the structure between years, a PERMANOVA with 999 permutations was applied (Anderson, 2001). The corals that made the greatest contribution to the dissimilarity between groups were defined by SIMPER analysis (Clarke, 1993). When making comparisons at the local and national level, differences derived from field evaluation methods were obviated. Thus, the comparison and interpretation are 
De este modo, la comparación e interpretación se limita a los valores de cobertura de las principales categorías del sustrato y la abundancia de especies coralinas.

\section{RESULTADOS}

\section{Caracterización histórica}

Entre 1989 y 2010, en las formaciones coralinas del archipiélago de San Bernardo el sustrato abiótico fue el componente más conspicuo. No obstante, entre 1989 y limited to the coverage values of the main categories of the substrate and the abundance of coral species.

\section{RESULTS}

\section{Historical characterization}

Between 1989 and 2010, in the coral formations of the San Bernardo archipelago, the abiotic substrate was the most conspicuous component. However, between 1989 and 2015 there was a decrease close to $45 \%$. During
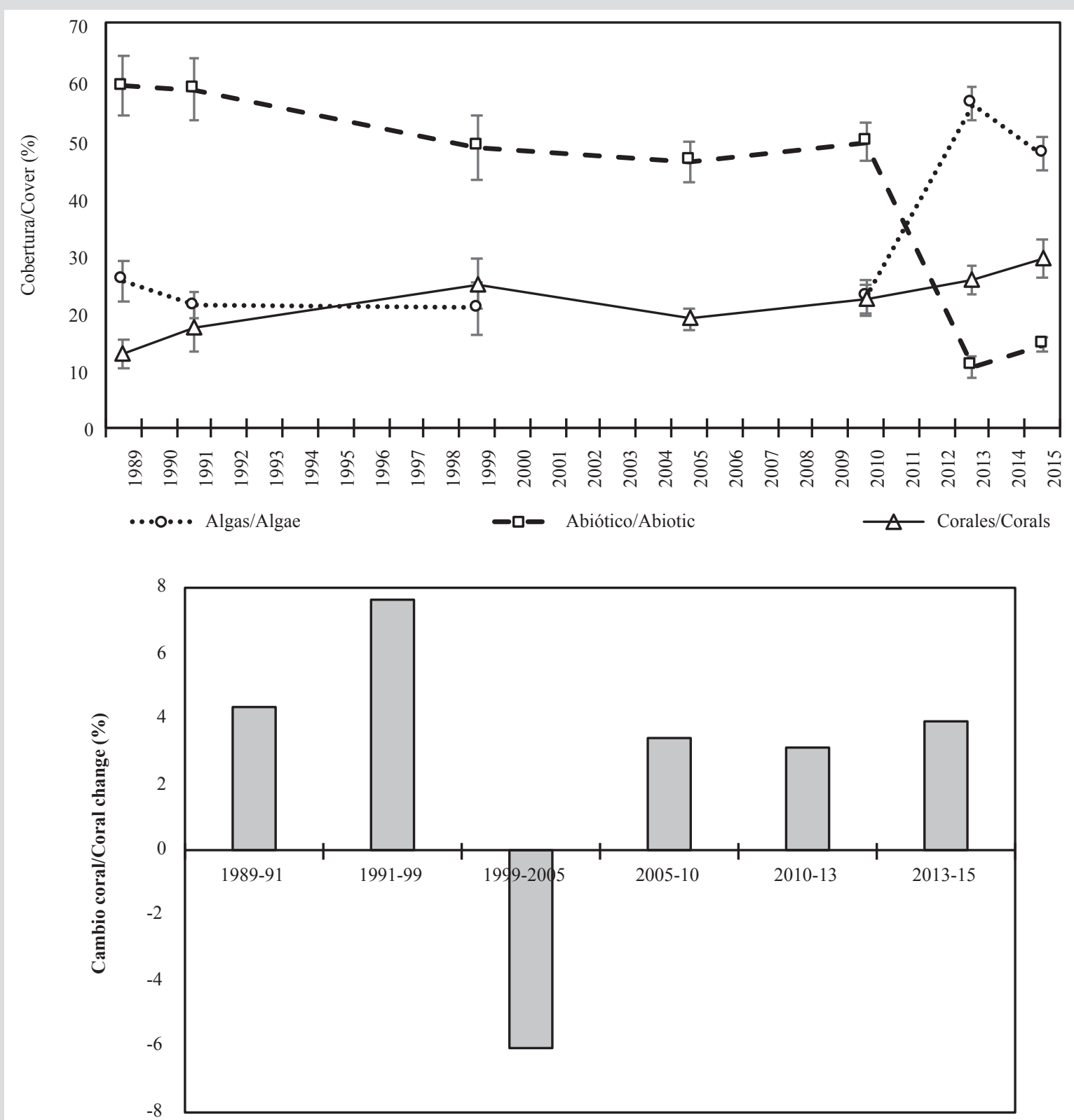

Figura 2. A) Cobertura promedio ( \pm error estándar) de coral, algas y sustrato abiótico. B) Tasa de cambio de la cobertura coralina entre 1989 y 2015 en el archipiélago de San Bernardo.
Figure 2. A) Average cover ( \pm standard error) of coral, algae, and abiotic substrate. B) The rate of change in coral cover between 1989 and 2015 in the San Bernardo archipelago. 
2015 se presentó un descenso cercano al $45 \%$. Durante la década de 1990 el componente algal presentó una reducción en su cobertura, siendo 1999 el único año en el que estuvo por debajo de los corales. Sin embargo, a partir del 2010 se registró un incremento, alcanzando un máximo en 2013, cuando se volvió el componente predominante.

En cuanto al componente coralino, entre 1989 y 1991 presentó baja cobertura (inferior a $20 \%$ ). A partir de 2005 se registró un incremento gradual en la cobertura que en 2015 fue cercana a $30 \%$. No obstante, en 2005 se observó una reducción de la cobertura en $6 \%$ con respecto a 1999 (Figura 2).

En la Tabla 1 se muestra la cobertura de cada taxón coralino registrado en cada uno de los años. Desde 1989 los corales que en general registraron una mayor abundancia relativa en el área de estudio fueron Orbicella spp. y Porites porites, seguidos de Agaricia spp., P. astreoides y Siderastrea spp. Se destaca la contribución histórica del género Orbicella que fue mayor a $35 \%$, así como su cobertura, la cual se incrementó en el tiempo. Adicionalmente, en el descenso de la cobertura del 2005, los géneros más afectados fueron Colpophyllia, Porites y Pseudodiporia. the $1990 \mathrm{~s}$, the algal component presented a reduction in its coverage, being 1999 the only year in which it was below the corals. However, as of 2010, there was an increase, reaching a maximum in 2013, when it became the predominant component.

Regarding the coral component, between 1989 and 1991 it presented low coverage (less than $20 \%$ ). Starting in 2005, there was a gradual increase in coverage, which in 2015 was close to $30 \%$. However, in 2005 there was a $6 \%$ reduction in coverage compared to 1999 (Figure 2).

Table 1 shows the coverage of each coral taxon recorded in each of the years. Since 1989, the corals that generally registered the highest relative abundance in the study area were Orbicella spp. and Porites porites, followed by Agaricia spp., P. astreoides and Siderastrea spp. The historical contribution of the Orbicella genus stands out, which was greater than $35 \%$, as well as its coverage, which increased over time. Additionally, in the decrease in coverage in 2005, the most affected genera were Colpophyllia, Porites, and Pseudodiporia.
Tabla 1. Porcentaje de cobertura media $(\bar{x})$ y error estándar (EE) de los corales en el archipiélago de San Bernardo entre 1989 y 2015.
Table 1. Percentage of mean coverage $(\bar{x})$ and standard error (SE) of corals in the San Bernardo archipelago between 1989 and 2015.

\begin{tabular}{|c|c|c|c|c|c|c|c|c|c|c|c|c|c|c|}
\hline \multirow{2}{*}{ Especie/Species } & \multicolumn{2}{|c|}{1989} & \multicolumn{2}{|c|}{1991} & \multicolumn{2}{|c|}{1999} & \multicolumn{2}{|c|}{2005} & \multicolumn{2}{|c|}{2010} & \multicolumn{2}{|c|}{2013} & \multicolumn{2}{|c|}{2015} \\
\hline & $\bar{x}$ & EE/SE & $\bar{x}$ & EE/SE & $\bar{x}$ & EE/SE & $\bar{x}$ & EE/SE & $\bar{x}$ & EE/SE & $\bar{x}$ & EE/SE & $\bar{x}$ & EE/SE \\
\hline Agaricia spp. & 0.53 & 0.26 & 0.92 & 0.37 & 1.30 & 0.93 & 1.00 & 0.46 & 1.85 & 1.06 & 2.37 & 0.97 & 4.16 & 1.71 \\
\hline Acropora cervicornis & 0.004 & 0.003 & 0.002 & 0.001 & 0.03 & 0.02 & - & - & 0.30 & 0.20 & 0.03 & 0.02 & 0.20 & 0.13 \\
\hline Acropora palmata & 0.01 & 0.01 & - & - & 0.002 & 0.001 & 0.03 & 0.02 & - & - & 1.04 & 0.42 & 0.28 & 0.10 \\
\hline Colpophyllia natans & 0.20 & 0.10 & 0.38 & 0.10 & 0.27 & 0.18 & 0.34 & 0.09 & 0.15 & 0.07 & 0.32 & 0.10 & 0.41 & 0.18 \\
\hline Dichocoenia stokesii & 0.04 & 0.01 & 0.10 & 0.03 & 0.06 & 0.03 & 0.05 & 0.02 & 0.01 & 0.01 & 0.04 & 0.03 & 0.14 & 0.05 \\
\hline Diploria labyrinthiformis & 0.24 & 0.09 & 0.30 & 0.11 & 0.22 & 0.11 & 0.17 & 0.06 & 0.16 & 0.06 & 0.22 & 0.08 & 0.47 & 0.19 \\
\hline Eusmilia fastigiata & 0.05 & 0.02 & 0.08 & 0.02 & 0.09 & 0.03 & 0.07 & 0.03 & 0.08 & 0.07 & 0.12 & 0.05 & 0.12 & 0.04 \\
\hline Favia fragum & 0.01 & 0.004 & 0.03 & 0.01 & 0.02 & 0.01 & 0.04 & 0.02 & 0.01 & 0.01 & 0.15 & 0.04 & 0.07 & 0.03 \\
\hline Helioseris cucullata & - & - & 0.01 & - & - & - & - & - & - & - & - & - & - & - \\
\hline Isophyllia rigida & 0.004 & 0.002 & 0.02 & 0.01 & 0.004 & 0.001 & 0.01 & 0.01 & 0.01 & 0.01 & 0.01 & 0.01 & - & - \\
\hline Isophyllia sinuosa & 0.02 & 0.01 & 0.010 & 0.003 & 0.05 & 0.02 & - & - & 0.004 & 0.001 & - & - & 0.03 & 0.02 \\
\hline Madracis auretenra & 0.10 & 0.04 & 0.14 & 0.10 & 0.02 & 0.01 & 0.04 & 0.02 & - & - & - & - & - & - \\
\hline
\end{tabular}




\begin{tabular}{|c|c|c|c|c|c|c|c|c|c|c|c|c|c|c|}
\hline \multirow{2}{*}{ Especie/Species } & \multicolumn{2}{|c|}{1989} & \multicolumn{2}{|c|}{1991} & \multicolumn{2}{|c|}{1999} & \multicolumn{2}{|c|}{2005} & \multicolumn{2}{|c|}{2010} & \multicolumn{2}{|c|}{2013} & \multicolumn{2}{|c|}{2015} \\
\hline & $\bar{x}$ & $\mathbf{E E} / \mathbf{S E}$ & $\bar{x}$ & EE/SE & $\bar{x}$ & EE/SE & $\bar{x}$ & EE/SE & $\bar{x}$ & EE/SE & $\bar{x}$ & EE/SE & $\bar{x}$ & EE/SE \\
\hline Madracis decactis & - & - & 0.03 & 0.01 & - & - & - & - & - & - & - & - & - & - \\
\hline Madracis pharensis & - & - & - & - & - & - & 0.09 & 0.01 & - & - & - & - & - & - \\
\hline Manicina areolata & 0.02 & 0.01 & 0.00 & 0.002 & - & - & 0.14 & 0.04 & - & - & 0.03 & 0.02 & - & - \\
\hline Meandrina meandrites & 0.02 & 0.01 & 0.01 & 0.01 & 0.08 & 0.04 & 0.09 & 0.03 & 0.08 & 0.03 & 0.10 & 0.04 & 0.23 & 0.09 \\
\hline Millepora alcicornis & 0.28 & 0.11 & 0.46 & 0.21 & 0.83 & 0.30 & 0.52 & 0.15 & 0.55 & 0.18 & 1.04 & 0.28 & 1.05 & 0.38 \\
\hline Millepora complanata & 0.08 & 0.07 & 0.04 & 0.03 & 0.17 & 0.11 & 0.60 & 0.34 & 0.17 & 0.09 & 2.32 & 0.76 & 1.76 & 0.39 \\
\hline Millepora squarrosa & 0.16 & 0.10 & 0.04 & 0.03 & 0.51 & 0.32 & - & - & 0.49 & 0.22 & - & - & - & - \\
\hline Montastraea cavernosa & 0.67 & 0.25 & 1.80 & 0.51 & 0.90 & 0.35 & 1.74 & 0.33 & 0.73 & 0.17 & 0.44 & 0.18 & 0.90 & 0.30 \\
\hline Mussa angulosa & - & - & 0.05 & - & - & - & - & - & - & - & 0.13 & - & - & - \\
\hline Mycetophyllia spp. & 0.01 & 0.01 & 0.02 & 0.01 & 0.01 & 0.01 & 0.01 & 0.01 & 0.01 & 0.01 & - & - & 0.02 & 0.01 \\
\hline Oculina difussa & - & - & 0.01 & - & - & - & - & - & - & - & - & - & - & - \\
\hline Orbicella spp. & 4.37 & 1.23 & 5.68 & 1.73 & 7.22 & 2.02 & 7.69 & 1.12 & 9.53 & 1.84 & 8.02 & 1.89 & 10.13 & 2.30 \\
\hline Porites astreoides & 1.59 & 0.79 & 0.73 & 0.33 & 1.67 & 0.40 & 1.96 & 0.39 & 1.64 & 0.35 & 2.05 & 0.46 & 2.23 & 0.54 \\
\hline Porites divaricata & - & - & - & - & - & - & - & - & - & - & 0.09 & 0.05 & 0.49 & 0.08 \\
\hline Porites porites & 3.60 & 1.31 & 5.96 & 4.99 & 11.04 & 4.36 & 2.24 & 0.87 & 5.89 & 1.71 & 4.27 & 1.27 & 3.34 & 1.13 \\
\hline Pseudodiploria clivosa & 0.01 & 0.01 & 0.002 & 0.002 & - & - & 0.01 & 0.01 & 0.03 & 0.01 & 0.21 & 0.09 & 0.54 & 0.24 \\
\hline Pseudodiploria strigosa & 0.31 & 0.14 & 0.32 & 0.12 & 0.28 & 0.13 & 0.40 & 0.20 & 0.12 & 0.05 & 0.67 & 0.26 & 0.96 & 0.27 \\
\hline Scolymia sp. & 0.01 & 0.002 & 0.01 & 0.01 & 0.002 & 0.001 & 0.01 & 0.01 & 0.01 & 0.001 & - & - & - & - \\
\hline Siderastrea spp. & 0.64 & 0.22 & 1.12 & 0.37 & 0.97 & 0.19 & 2.02 & 0.31 & 1.20 & 0.23 & 3.19 & 1.56 & 3.23 & 1.16 \\
\hline Solenastrea bournoni & - & - & - & - & - & - & - & - & - & - & 0.07 & 0.02 & 0.22 & 0.05 \\
\hline Stephanocoenia intersepta & 0.02 & 0.01 & 0.05 & 0.02 & 0.01 & 0.004 & 0.14 & 0.03 & 0.03 & 0.01 & 0.02 & 0.01 & 0.18 & 0.09 \\
\hline
\end{tabular}

A nivel espacial, se evidenció un patrón de mayor cobertura de algas y, por lo general, menor cobertura de coral, en los sectores que se encuentran más cerca del continente como Palma (C-18) y Cabruna (C-19), especialmente en 2015 (Figura 3). En estas estaciones fueron abundantes los corales de los géneros Agaricia y Porites. Por el contrario, las estaciones C-6 (Múcura), C-11 (Tintipán) y C-14 (Mangle) estuvieron entre los sitios que históricamente registraron mayores valores de cobertura coralina, en las que el género Orbicella fue el más abundante (Tablas 1 y 2).
At a spatial level, a pattern of greater algae cover, and generally less coral cover, as evidenced in sectors that are closer to the continent such as Palma (C-18) and Cabruna (C-19), especially in 2015 (Figure 3). Corals of the genera Agaricia and Porites were abundant at these stations. On the contrary, stations C-6 (Múcura), C-11 (Tintipán) and C-14 (Mangle), were among the sites that historically registered the highest values of coral cover, in which the genus Orbicella was the most abundant (Tables 1 and 2). 


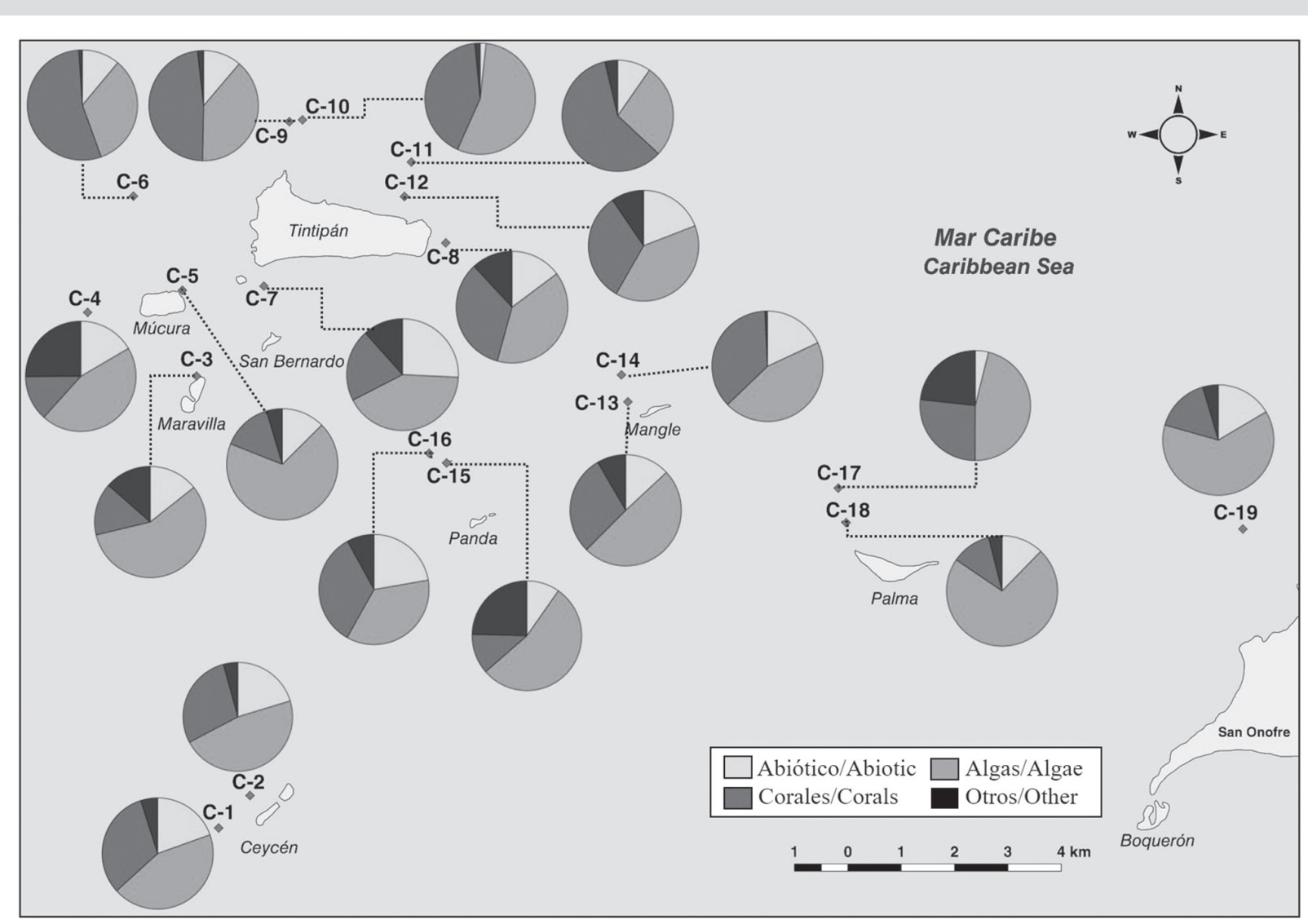

Figura 3. Cobertura de los componentes del sustrato (\%) en cada una de las estaciones en el área del archipiélago de San Bernardo en 2015. C-1 y C-2 corresponden al sector Ceycen; C-3 a Maravilla; C-4, C-5 y C-6 a Múcura; C-7, C-8, C-9, C-10, C-11 y C-12 a Tintipán; C-13 y C-14 a Mangle; C-15 y C-16 a Panda; C-17 y C-18 a Palma, y C-19 a Cabruna.
Figure 3. Coverage of the substrate components (\%) in each of the stations in the San Bernardo archipelago area in 2015. C-1 and C-2 correspond to the Ceycen sector; C-3 to Maravilla; C-4, C-5, and C-6 to Múcura; C-7, C-8, C-9, C-10, C-11, and C-12 to Tintipán; C-13 and C-14 to Mangle; C-15 and C-16 to Panda; C-17 and C-18 to Palma, and C-19 to Cabruna.
Tabla 2. Cobertura relativa de coral (\%) y profundidad (m) en cada una de las estaciones evaluadas en el archipiélago de San Bernardo entre 1989 y 2015.
Table 2. Relative coral cover (\%) and depth (m) in each of the stations evaluated in the San Bernardo archipelago between 1989 and 2015.

\begin{tabular}{|c|c|c|c|c|c|c|c|c|}
\hline Estación/Station & Profundidad/Depth (m) & 1989 & 1991 & 1999 & 2005 & 2010 & 2013 & 2015 \\
\hline $\mathrm{C}-1$ & 5.0 & 5.5 & 5.8 & 9.7 & 8.5 & 18.8 & 41.6 & 32.5 \\
\hline $\mathrm{C}-2$ & 4.0 & - & 17.5 & 12.7 & 20.6 & 34.3 & 32.2 & 28.5 \\
\hline $\mathrm{C}-3$ & 6.0 & 5.8 & - & 9.0 & 15.7 & 16.4 & 13.7 & 15.2 \\
\hline C-4 & 6.5 & - & 9.7 & 18.2 & 12.4 & 10.8 & 27.7 & 13.3 \\
\hline $\mathrm{C}-5$ & 4.5 & 7.8 & - & 75.1 & 13.1 & 18.3 & 13.5 & 14.4 \\
\hline C-6 & 5.5 & 26.4 & - & 42.7 & 35.7 & 25.0 & 37.8 & 54.5 \\
\hline $\mathrm{C}-7$ & 4.0 & - & 11.8 & 10.2 & 13.0 & 23.2 & 15.6 & 20.9 \\
\hline $\mathrm{C}-8$ & 8.0 & 7.9 & 10.6 & 6.0 & 21.3 & 18.7 & 19.8 & 33.8 \\
\hline C-9 & 7.0 & 7.9 & 10.6 & 6.0 & 21.3 & 18.7 & 19.8 & 33.8 \\
\hline C-10 & 8.3 & 11.3 & - & 18.1 & 28.5 & 27.3 & 39.3 & 41.7 \\
\hline
\end{tabular}




\begin{tabular}{|c|c|c|c|c|c|c|c|c|c|c|}
\hline Estación/Station & Profundidad/Depth (m) & $\mathbf{1 9 8 9}$ & $\mathbf{1 9 9 1}$ & $\mathbf{1 9 9 9}$ & $\mathbf{2 0 0 5}$ & $\mathbf{2 0 1 0}$ & $\mathbf{2 0 1 3}$ & $\mathbf{2 0 1 5}$ \\
\hline C-11 & 6.0 & - & 18.7 & 29.3 & 17.1 & 40.0 & 38.9 & 59.3 \\
\hline C-12 & 8.0 & - & - & - & 26.3 & 18.0 & 21.8 & 32.2 \\
\hline C-13 & 4.0 & - & 12.1 & 18.7 & 13.4 & 11.6 & 33.5 & 29.1 \\
\hline C-14 & 5.5 & 26.2 & 29.5 & 20.0 & 20.0 & 37.3 & 41.5 & 36.5 \\
\hline C-15 & 4.0 & 10.8 & - & 45.1 & 25.6 & 9.5 & 8.9 & 11.8 \\
\hline C-16 & 11.2 & - & 7.7 & 8.8 & 8.2 & 5.8 & 23.5 \\
\hline C-17 & 9.5 & 5.1 & - & 16.3 & 10.3 & 24.3 & 23.2 \\
\hline C-18 & 5.5 & - & 49.3 & 48.1 & 32.4 & 40.7 & 11.1 \\
\hline C-19 & 6.0 & 13.6 & - & 30.8 & 22.0 & 16.7 & 11.5 \\
\hline
\end{tabular}

Según el índice de Shannon-Wiener (Ln), la diversidad promedio históricamente ha sido mayor a 1,6 nits, exceptuando los años 1999 y 2010. La baja diversidad en estos dos años se relaciona con una menor uniformidad, ya que fueron los únicos periodos en que este último índice fue inferior a 0,6. La mayor diversidad en 2015 y 2005 fue más por efecto de una mayor uniformidad $\left(\mathrm{J}_{\overline{\mathrm{x}}}>0,69\right)$, mientras en 1989 y 1991 fue más resultado de una mayor riqueza $\left(\mathrm{S}_{\overline{\mathrm{x}}}>13\right.$; Figura 4$)$.

La estructura (composición y abundancia) del ensamblaje coralino presentó diferencias significativas temporales (PERMANOVA, $\mathrm{F}_{(6,116)}=2,19 ; \mathrm{P}=0,001$ ). Pruebas de $t$ por parejas indicaron que particularmente 2015 y 2013 fueron diferentes de los otros años $(\mathrm{P}<0,05)$, mas no entre ellos, y que además 1989 presentó diferencias significativas con 2005 (Tabla 3), como evidencia la gráfica de promedios Bootstrap (Figura 5).

Tabla 3. Comparación entre años de los resultados estadísticos de la prueba t (probabilidad), usando 999 permutaciones. El asterisco indica donde se registraron diferencias significativas.
According to the Shannon-Wiener (Ln) index, the average diversity historically has been greater than 1.6 nits, except for the years 1999 and 2010. The low diversity in these two years is related to less uniformity since they were the only periods in which the latter index was less than 0.6. The greater diversity in 2015 and 2005 was more due to greater uniformity $\left(\mathrm{J}_{\overline{\mathrm{x}}}>0,69\right)$, while in 1989 and 1991 it was more the result of greater wealth $\left(\mathrm{S}_{\overline{\mathrm{x}}}>13\right.$; Figure 4).

The structure (composition and abundance) of the coral assemblage showed significant temporal differences (PERMANOVA, $\mathrm{F}_{(6,116)}=2.19 ; \mathrm{P}=0.001$ ). Pairwise t-tests indicated that particularly 2015 and 2013 were different from the other years $(\mathrm{P}<0.05)$, but not between them, and that 1989 also presented significant differences with 2005 (Table 3), as evidenced by the graph of averages Bootstrap (Figure 5).

Table 3. Comparison between years of the statistical results of the t-test (probability), using 999 permutations. The asterisk indicates where significant differences were recorded.

\begin{tabular}{|c|c|c|c|c|c|c|}
\hline Años/Years & 1991 & 1999 & 2005 & 2010 & 2013 & 2015 \\
\hline 1989 & $0.86(0.56)$ & $1.01(0.36)$ & $1.71(0.02)^{*}$ & $1.41(0.08)$ & $1.65(0.01)^{*}$ & $1.78(0.01)^{*}$ \\
1991 & - & $1.13(0.22)$ & $1.25(0.13)$ & $1.39(0.10)$ & $1.59(0.01)^{*}$ & $1.56(0.02)^{*}$ \\
1999 & - & - & $1.56(0.05)^{*}$ & $0.77(0.64)$ & $1.39(0.05)^{*}$ & $1.52(0.04)^{*}$ \\
2005 & - & - & - & $1.49(0.06)$ & $1.96\left(3.0 \times 10^{-3}\right)^{*}$ & $1.95\left(2.0 \times 10^{-3}\right)^{*}$ \\
2010 & - & - & - & - & $1.66(0.02)^{*}$ & $1.61(0.03)^{*}$ \\
2013 & - & - & - & - & - & \\
\hline
\end{tabular}



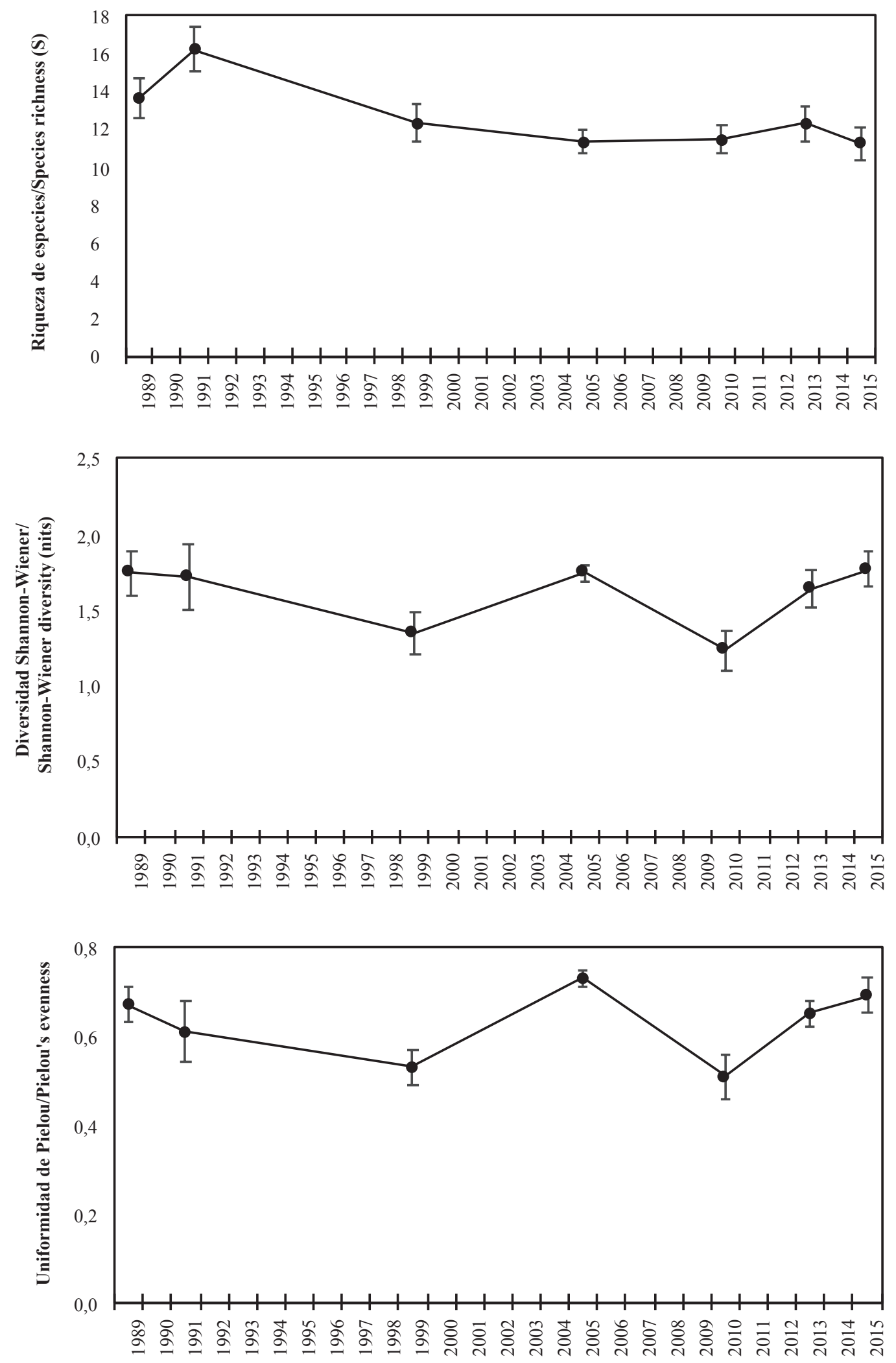

Figura 4. Medidas de diversidad promedio ( \pm error estándar) del ensamblaje coralino en el archipiélago de San Bernardo entre 1989 y 2015. A) Riqueza de especies. B) Diversidad de Shannon-Wiener (nits). C) Uniformidad de Pielou.
Figure 4. Average diversity measurements ( \pm standard error) of the coral assemblage in the San Bernardo archipelago between 1989 and 2015. A) Species richness. B) Shannon-Wiener diversity (nits). C) Pielou uniformity. 


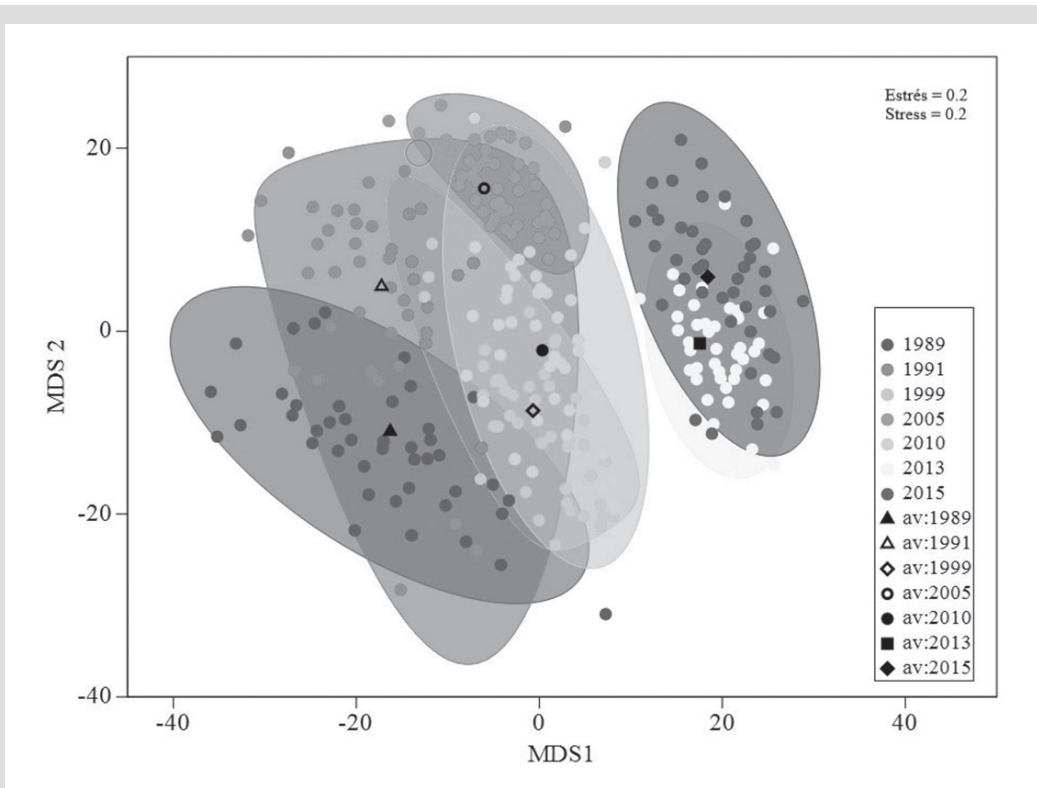

Figura 5. Gráfica bivariada de promedios Bootstrap del ensamblaje coralino entre años. Los círculos indican el promedio de cada repetición (transectos en un año), los marcadores negros el promedio general de cada año y los límites de las figuras geométricas corresponden a la región Bootstrap de $95 \%$ de confianza.
Figure 5. Bivariate graph of Bootstrap averages of the coral assemblage between years. The circles indicate the average of each repetition (transects in a year), the black markers represent the general average of each year and the limits of the geometric. Figures correspond to the Bootstrap region of $95 \%$ confidence.
La prueba SIMPER reveló que los corales Orbicella spp., P. porites, Agaricia spp., Siderastrea spp. y Millepora complanata contribuyeron con al menos $70 \%$ de la disimilitud temporal (Tabla 4). Estas especies presentaron mayor cobertura en 2013 y 2015. En el caso de P. porites, aunque su contribución relativa a la disimilitud temporal del ensamblaje coralino varió entre $21,2 \%$ (1999) y $12,8 \%$ (2013), su cobertura tiende a ser cíclica después del 2005, momento que registró una reducción cercana a $9 \%$ (Tabla 1).
The SIMPER test revealed that the corals Orbicella spp., P. porites, Agaricia spp., Siderastrea spp. and Millepora complanata contributed at least $70 \%$ of the temporal dissimilarity (Table 4). These species presented greater coverage in 2013 and 2015. In the case of P. porites, although its relative contribution to the temporal dissimilarity of the coral assembly varied between $21.2 \%$ (1999) and $12.8 \%$ (2013), its coverage tends to be cyclical after 2005, when it registered a reduction close to $9 \%$ (Table 1).
Tabla 4. Taxa que más contribuyen a la disimilitud del ensamblaje coralino entre años con base en el análisis SIMPER a un corte de contribución acumulada de mínimo el 70 \%. El primer valor corresponde a la disimilitud promedio (\%), el segundo a la consistencia (disimilitud/desviación estándar) y el tercero a la contribución relativa (\%) de cada taxón.
Table 4. Taxa that most contribute to the dissimilarity of the coral assemblage between years based on the SIMPER analysis at a cumulative contribution cutoff of at least $70 \%$. The first value corresponds to the average dissimilarity $(\%)$, the second to the consistency (dissimilarity/standard deviation) and the third to the relative contribution (\%) of each taxon.

\begin{tabular}{|c|c|c|c|c|c|c|c|}
\hline Años/Years & $\begin{array}{l}\text { Porites } \\
\text { porites }\end{array}$ & $\begin{array}{l}\text { Orbicella } \\
\text { spp. }\end{array}$ & $\begin{array}{c}\text { Montastraea } \\
\text { cavernosa }\end{array}$ & $\begin{array}{c}\text { Porites } \\
\text { astreoides }\end{array}$ & $\begin{array}{l}\text { Siderastrea } \\
\text { spp. }\end{array}$ & $\begin{array}{l}\text { Agaricia } \\
\text { spp. }\end{array}$ & $\begin{array}{c}\text { Millepora } \\
\text { complanata }\end{array}$ \\
\hline 1989 vs. 1991 & $\begin{array}{c}17.77 ; 0.79 \\
28.45\end{array}$ & $\begin{array}{c}16.69 ; 1.33 ; \\
26.73\end{array}$ & $5.97 ; 1.04 ; 9.55$ & $4.98 ; 0.73 ; 7.97$ & & & \\
\hline 1989 vs. 1999 & $\begin{array}{l}22.57 ; 1.00 \\
34.63\end{array}$ & $\begin{array}{c}\text { 17.38; } 1.18 ; \\
26.65\end{array}$ & & $6.09 ; 0.96 ; 9.35$ & & & \\
\hline 1991 vs. 1999 & $\begin{array}{c}23.37 ; 0.88 ; \\
35.60\end{array}$ & $\begin{array}{c}17.05 ; 1.23 \\
25.98\end{array}$ & $5.00 ; 1.03 ; 7.61$ & $4.16 ; 0.91 ; 6.33$ & & & \\
\hline 1989 vs. 2005 & $\begin{array}{c}\text { 11.58; 1.04; } \\
19.39\end{array}$ & $\begin{array}{l}17.46 ; 1.52 \\
29.25\end{array}$ & $5.06 ; 1.23 ; 8.48$ & $6.68 ; 1.12 ; 11.19$ & $5.59 ; 1.34 ; 9.37$ & & \\
\hline
\end{tabular}




\begin{tabular}{|c|c|c|c|c|c|c|c|}
\hline Años/Years & $\begin{array}{l}\text { Porites } \\
\text { porites }\end{array}$ & $\begin{array}{l}\text { Orbicella } \\
\text { spp. }\end{array}$ & $\begin{array}{c}\text { Montastraea } \\
\text { cavernosa }\end{array}$ & $\begin{array}{c}\text { Porites } \\
\text { astreoides }\end{array}$ & $\begin{array}{l}\text { Siderastrea } \\
\text { spp. }\end{array}$ & $\begin{array}{l}\text { Agaricia } \\
\text { spp. }\end{array}$ & $\begin{array}{l}\text { Millepora } \\
\text { complanata }\end{array}$ \\
\hline 1991 vs. 2005 & $\begin{array}{l}12.12 ; 0.59 ; \\
21.56\end{array}$ & $\begin{array}{c}16.23 ; 1.49 \\
28.87\end{array}$ & $5.12 ; 1.21 ; 9.11$ & $4.6 ; 01.02 ; 8.18$ & $4.91 ; 1.29 ; 8.73$ & & \\
\hline 1999 vs. 2005 & $\begin{array}{l}\text { 18.52; } 0.87 \\
\quad 30.89\end{array}$ & $\begin{array}{c}15.85 ; 1.36 \\
26.43\end{array}$ & $4.37 ; 1.14 ; 7.30$ & $4.46 ; 1.01 ; 7.43$ & & & \\
\hline 1989 vs. 2010 & $17.5 ; 1.02 ; 27.46$ & $\begin{array}{c}21.89 ; 1.19 ; \\
34.35\end{array}$ & & $5.70 ; 1.02 ; 8.95$ & & & \\
\hline 1991 vs. 2010 & $\begin{array}{c}19.02 ; 0.84 \\
29.78\end{array}$ & $\begin{array}{l}20.16 ; 1.23 ; \\
\quad 31.56\end{array}$ & $4.21 ; 1.01 ; 6.60$ & & & $5.38 ; 0.62 ; 8.42$ & \\
\hline 1999 vs. 2010 & $\begin{array}{l}21.44 ; 1.03 ; \\
\quad 34.38\end{array}$ & $\begin{array}{c}19.16 ; 1.28 \\
30.73\end{array}$ & & & & $4.71 ; 0.53 ; 7.56$ & \\
\hline 2005 vs. 2010 & $13.72 ; 0.9 ; 24.62$ & $\begin{array}{c}\text { 17.37; } 1.33 ; \\
31.17\end{array}$ & & $3.94 ; 0.98 ; 7.07$ & & $4.91 ; 0.60 ; 8.81$ & \\
\hline 1989 vs. 2013 & $\begin{array}{c}12.90 ; 1.02 \\
18.27\end{array}$ & $\begin{array}{l}\text { 17.54; } 1.25 \\
24.85\end{array}$ & & $6.57 ; 1.06 ; 9.31$ & $6.78 ; 0.60 ; 9.6$ & $6.63 ; 0.61 ; 9.39$ & \\
\hline 1991 vs. 2013 & $\begin{array}{c}\text { 14.44; } 0.72 \\
20.33\end{array}$ & $\begin{array}{c}17.66 ; 1.25 \\
24.86\end{array}$ & & & $6.60 ; 0.64 ; 9.29$ & $6.64 ; 0.66 ; 9.35$ & $5.78 ; 0.59 ; 8.13$ \\
\hline 1999 vs. 2013 & $\begin{array}{l}\text { 18.71; } 0.93 ; \\
26.69\end{array}$ & $\begin{array}{l}\text { 17.54; } 1.20 \\
25.03\end{array}$ & & & $5.57 ; 0.60 ; 7.95$ & $6.25 ; 0.63 ; 8.91$ & $4.96 ; 0.59 ; 7.07$ \\
\hline 2005 vs. 2013 & $9.42 ; 0.90 ; 14.78$ & $\begin{array}{l}\text { 17.29; } 1.40 \\
27.12\end{array}$ & & & $6.91 ; 0.77 ; 10.84$ & $6.38 ; 0.68 ; 10.01$ & $5.57 ; 0.66 ; 8.74$ \\
\hline 2010 vs. 2013 & $\begin{array}{l}\text { 14.22; } 0.97 ; \\
21.16\end{array}$ & $\begin{array}{l}\text { 19.78; } 1.21 \\
29.42\end{array}$ & & & $5.75 ; 0.64 ; 8.55$ & $7.25 ; 0.69 ; 10.78$ & $4.90 ; 0.61 ; 7.29$ \\
\hline 1989 vs. 2015 & $\begin{array}{c}\text { 10.83; 0.96; } \\
15.16\end{array}$ & $\begin{array}{c}19.07 ; 1.34 \\
26.69\end{array}$ & & $5.71 ; 1.03 ; 7.99$ & $7.94 ; 0.69 ; 11.11$ & $8.20 ; 0.51 ; 11.47$ & \\
\hline 1991 vs. 2015 & $\begin{array}{c}12.30 ; 0.65 \\
17.50\end{array}$ & $\begin{array}{c}18.71 ; 1.41 \\
26.63\end{array}$ & & & $7.29 ; 0.70 ; 10.37$ & $8.01 ; 0.55 ; 11.41$ & $4.32 ; 0.83 ; 6.15$ \\
\hline 1999 vs. 2015 & $\begin{array}{c}16.69 ; 0.88 ; \\
23.78\end{array}$ & $\begin{array}{l}18.15 ; 1.36 \\
25.85\end{array}$ & & $4.24 ; 1.05 ; 6.04$ & $6.24 ; 0.67 ; 8.89$ & $7.59 ; 0.55 ; 10.82$ & \\
\hline 2005 vs. 2015 & $7.80 ; 0.83 ; 12.32$ & $\begin{array}{c}18.03 ; 1.70 \\
28.49\end{array}$ & & $4.37 ; 1.09 ; 6.91$ & $6.77 ; 0.77 ; 10.7$ & $7.78 ; 0.57 ; 12.29$ & \\
\hline 2010 vs. 2015 & $\begin{array}{c}12.29 ; 0.92 ; \\
18.47\end{array}$ & $\begin{array}{c}19.22 ; 1.36 \\
28.89\end{array}$ & & $4.01 ; 1.09 ; 6.03$ & $6.26 ; 0.69 ; 9.41$ & $8.43 ; 0.61 ; 12.67$ & \\
\hline 2013 vs. 2015 & $9.06 ; 0.88 ; 13.46$ & $\begin{array}{c}18.07 ; 1.31 ; \\
26.82\end{array}$ & & & $8.23 ; 0.74 ; 12.21$ & $9.00 ; 0.65 ; 13.36$ & $5.24 ; 0.85 ; 7.77$ \\
\hline
\end{tabular}

En cuanto a la presencia de signos de deterioro coralino, en 2015 se observó blanqueamiento en todas las estaciones, excepto en C-7. Las especies más afectadas fueron Orbicella annularis, O. faveolata, Agaricia tenuifolia y Siderastrea siderea (Tabla 5) y en todos los casos el blanqueamiento afectó las colonias de forma parcial. Se
Regarding the presence of signs of coral deterioration, in 2015 bleaching was observed in all stations, except in C-7. The most affected species were Orbicella annularis, O. faveolata, Agaricia tenuifolia, and Siderastrea siderea (Table 5), and in all cases, the bleaching partially affected the colonies. White plague, 
registraron las enfermedades de plaga blanca, banda negra y lunares oscuros; esta última enfermedad fue la más frecuente (presente en 13 estaciones; Figura 6) y afectó principalmente al coral $S$. siderea. black band, and dark mole diseases were recorded; this last disease was the most frequent (present in 13 stations; Figure 6) and mainly affected $S$. siderea coral.
Tabla 5. Especies de coral observadas con blanqueamiento en las estaciones evaluadas en el archipiélago de San Bernardo en 2015.
Table 5. Coral species observed with bleaching in the stations evaluated in the San Bernardo archipelago in 2015.

\begin{tabular}{|c|c|c|c|c|c|c|c|c|c|c|c|c|c|c|c|c|c|c|c|c|}
\hline & $\bar{J}$ & ن̃ & $\tilde{u}$ & ঠ் & ర̊ & نَ & U் & ن் & iे & $\frac{\theta}{\bar{J}}$ & $\overline{\bar{u}}$ & $\frac{\pi}{0}$ & $\frac{m}{U}$ & $\frac{\vec{J}}{\vec{u}}$ & $\frac{n}{0}$ & $\frac{0}{0}$ & $\frac{5}{0}$ & $\frac{\infty}{\bar{J}}$ & $\frac{\theta}{\vec{u}}$ & 㡎 \\
\hline Acropora palmata & $\mathrm{X}$ & & & & & & & & & & & & $\mathrm{X}$ & & & & & & & 2 \\
\hline Agaricia tenuifolia & & & $\mathrm{X}$ & & $\mathrm{X}$ & & & & & $\mathrm{X}$ & $\mathrm{X}$ & & & $\mathrm{X}$ & & & $\mathrm{X}$ & & & 6 \\
\hline Colpophyllia natans & & & & & & & & & & & & & & & & $\mathrm{X}$ & & & & 1 \\
\hline Helioseris cucullata & & & & & & $\mathrm{X}$ & & & & & & & & & & & & & & 1 \\
\hline Millepora alcicornis & & & & & & & & & & & & & & & & & & $\mathrm{X}$ & & 1 \\
\hline Millepora complanata & $\mathrm{X}$ & & & & & & & & & & & & & & & & & $\mathrm{X}$ & $\mathrm{X}$ & 3 \\
\hline Montastraea cavernosa & & & & & & & & $\mathrm{X}$ & & & & & & & & & & & & 1 \\
\hline Orbicella annularis & & $\mathrm{X}$ & & & & & & $\mathrm{X}$ & $\mathrm{X}$ & $\mathrm{X}$ & & $\mathrm{X}$ & & $\mathrm{X}$ & & & & & $\mathrm{X}$ & 7 \\
\hline Orbicella faveolata & & $\mathrm{X}$ & & & & $\mathrm{X}$ & & & & & $\mathrm{X}$ & $\mathrm{X}$ & & $\mathrm{X}$ & & $\mathrm{X}$ & & & & 6 \\
\hline Pseudodiploria clivosa & & & & & & & & & & & & & $\mathrm{X}$ & & & & & & & 1 \\
\hline Pseudodiploria strigosa & & & & & & & & & & & & & & & $\mathrm{X}$ & & & & & 1 \\
\hline Porites astreoides & & & & & & & & & & & & & & $\mathrm{X}$ & & & & & $\mathrm{X}$ & 2 \\
\hline Porites porites & & & $\mathrm{X}$ & & & & & & & & & & & & & & & & $\mathrm{X}$ & 2 \\
\hline Siderastrea radians & & & & & & & & & & & & & $\mathrm{X}$ & & & & & & & 1 \\
\hline Siderastrea siderea & $X$ & $\mathrm{X}$ & $\mathrm{X}$ & $\mathrm{X}$ & & & & & & & & $\mathrm{X}$ & & & & & & & $\mathrm{X}$ & 6 \\
\hline Total especies/Total species & 3 & 3 & 3 & 1 & 1 & 2 & 0 & 2 & 1 & 2 & 2 & 3 & 3 & 4 & 1 & 2 & 1 & 2 & 5 & \\
\hline
\end{tabular}

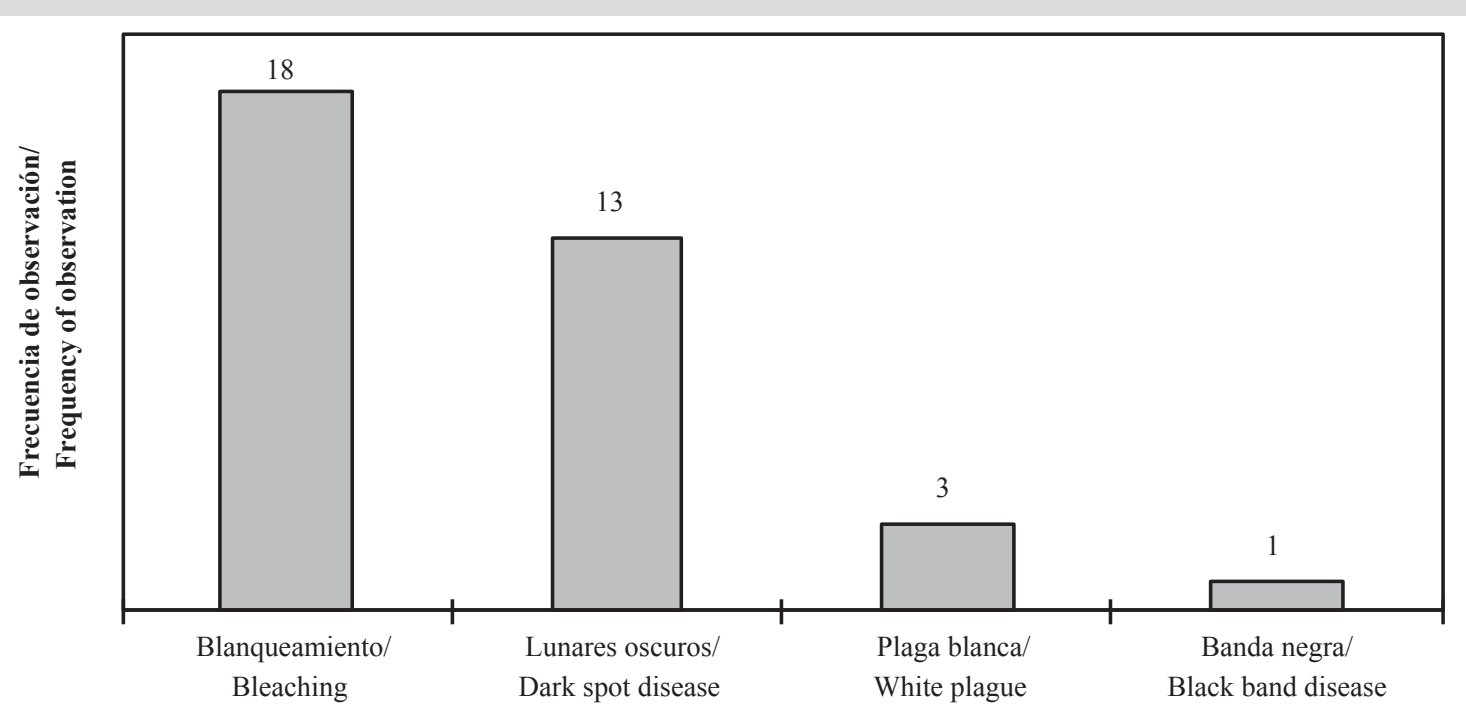

Figura 6. Cantidad de estaciones con la presencia de enfermedades coralinas y blanqueamiento en el archipiélago de San Bernardo en 2015.
Figure 6. Number of stations with the presence of coral diseases and bleaching in the San Bernardo archipelago in 2015. 
En la Figura 7 se muestra la temperatura superficial del mar (TSM) diaria durante 2015 en relación con el umbral de tolerancia al blanqueamiento de $29,4^{\circ} \mathrm{C}$. A partir de junio se registraron temperaturas que superaron el umbral y los valores más altos $\left(30,6{ }^{\circ} \mathrm{C}\right)$ se presentaron en julio y septiembre. Durante el monitoreo en agosto de 2015, se registró un DHW de $1,5^{\circ} \mathrm{C}$-semanas, que indica que los corales estuvieron experimentado un bajo estrés térmico. Posteriormente, entre octubre y diciembre se registraron valores de DWH entre 4 y $8{ }^{\circ} \mathrm{C}$-semanas, indicando un estrés térmico alto que puede ocasionar un blanqueamiento significativo.
Figure 7 shows the daily sea surface temperature (SST) during 2015 in relation to the whitening tolerance threshold of $29.4{ }^{\circ} \mathrm{C}$. Starting in June, temperatures that exceeded the threshold were recorded and the highest values $\left(30.6{ }^{\circ} \mathrm{C}\right)$ were recorded in July and September. During monitoring in August 2015, a DHW of $1.5^{\circ} \mathrm{C}$-weeks was recorded, indicating that the corals were experiencing low heat stress. Subsequently, between October and December, DWH values were recorded between 4 and $8{ }^{\circ} \mathrm{C}$-weeks, indicating high thermal stress that can cause significant bleaching.

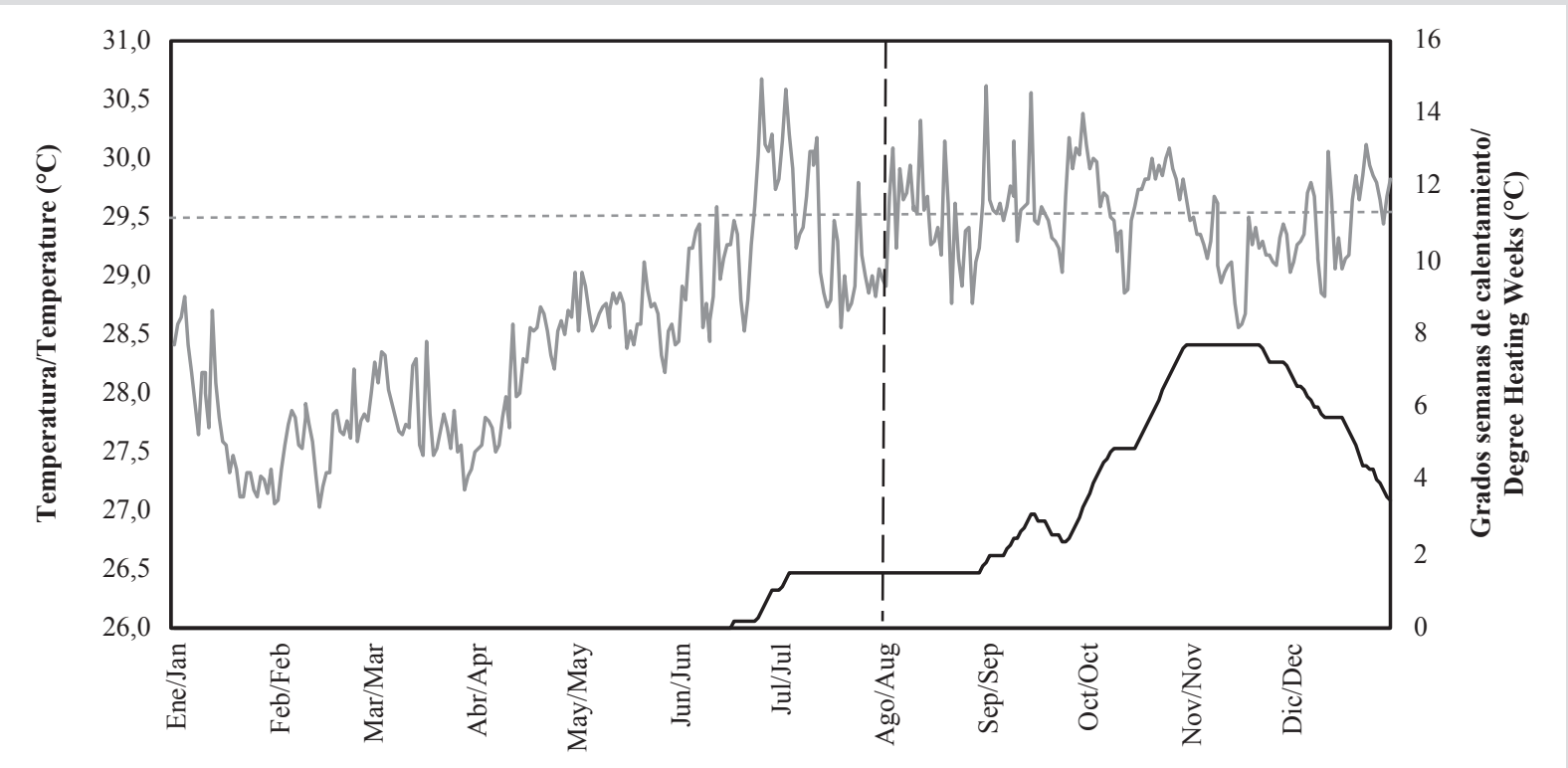

Figura 7. Temperatura superficial del mar $\left({ }^{\circ} \mathrm{C}\right.$, línea gris $)$ y estrés térmico DHW $\left({ }^{\circ} \mathrm{C}\right.$-semanas, línea negra continua) entre enero y diciembre de 2015. Datos satelitales de la NOAA para Colombia, resolución $5 \mathrm{~km}$ (NOAA Coral Reef Watch, 2020). La línea roja punteada indica el umbral de tolerancia al blanqueamiento de la $\operatorname{NOAA}\left(29,4^{\circ} \mathrm{C}\right)$. La línea punteada vertical indica el momento en que fue realizado el seguimiento.
Figure 7. Sea surface temperature $\left({ }^{\circ} \mathrm{C}\right.$, gray line) and $\mathrm{DHW}$ thermal stress $\left({ }^{\circ} \mathrm{C}\right.$-weeks, solid black line) between January and December 2015. NOAA satellite data for Colombia, resolution $5 \mathrm{~km}$ (NOAA Coral Reef Watch, 2020). The red dotted line indicates the NOAA whitening tolerance threshold $\left(29.4{ }^{\circ} \mathrm{C}\right)$. The vertical dotted line indicates the time when the follow-up was performed.

\section{DISCUSIÓN}

Desde la década de 1980, las formaciones coralinas del Caribe colombiano, incluyendo las del Parque Nacional Natural Corales del Rosario y San Bernardo (PNNCRSB), han presentado cambios en la dominancia de los componentes bentónicos, composición coralina, mortandades masivas de organismos, disminución de la cobertura coralina, eventos de blanqueamiento e incremento de la prevalencia de enfermedades (Werding y Sánchez, 1979; Duque y Gómez, 1983; Alvarado et al., 1986, 2011; Cendales et al., 2002; Garzón-Ferreira y Díaz, 2003; Rodríguez-Ramírez et al., 2010; Jackson et al., 2014).

\section{DISCUSSION}

Since the 1980s, the coral formations of the Colombian Caribbean, including those of the Corales del Rosario and San Bernardo National Natural Park (PNNCRSB), have presented changes in the dominance of benthic components, coral composition, mass mortality of organisms, decrease of coral cover, bleaching events and increased disease prevalence (Werding and Sánchez, 1979; Duque and Gómez, 1983; Alvarado et al., 1986, 2011; Cendales et al., 2002; Garzón-Ferreira and Díaz, 2003; Rodríguez-Ramírez et al., 2010; Jackson et al., 2014). 
En el PNNCRSB y específicamente en las islas de San Bernardo se han identificado como principales amenazas la pesca sobre los recursos hidrobiológicos (Martínez-Viloria et al., 2011), la actividad turística (Mendoza et al., 2011), el uso de material biológico para elaboración de artesanías y recuerdos (Ordosgoitia y ZarzaGonzález; 2011) y el blanqueamiento coralino (GómezCampo et al., 2011).

Otras amenazas locales que se pueden sumar son la posible influencia de los sedimentos y el agua dulce provenientes del río Sinú y los hidrocarburos provenientes del tránsito de embarcaciones turísticas y derrames accidentales por las actividades asociadas al terminal marítimo de Coveñas. La influencia de agua dulce se evidencia en las concentraciones de alcalinidad tanto en época lluviosa (107,5 a 116,6 mg/L) como en época seca (119,2 a 130,8 mg/L) de 2013 en San Bernardo (Hyser, 2014). Estos valores están por debajo de los límites ideales $(175-200 \mathrm{mg} / \mathrm{L})$ e incluso aceptables (124-225 mg/L) establecidos para el cultivo de corales (Borneman, 2008), lo cual limita la calcificación y disminuye la tasa de crecimiento y la densidad esquelética (Borneman, 2008; Hurtado, 2015).

En el caso de los hidrocarburos, los resultados de Betancourt-Portela et al. (2011), que hacen parte del seguimiento que realiza la REDCAM de Invemar, encontraron concentraciones por debajo del nivel de referencia internacional de $10 \mathrm{mg} / \mathrm{L}$ en el PNNCRSB. Asimismo, este documento mostró que desde 2003 todos los muestreos han registrado un bajo nivel de contaminación que no representa riesgo para los organismos acuáticos. Tampoco hay evidencia que los derrames que se han producido en el golfo de Morrosquillo han llegado a afectar las formaciones coralinas del archipiélago de San Bernardo (Carsucre, 2014).

En el archipiélago de San Bernardo, entre 1989 y 1991 se registró una baja cobertura coralina $(<20 \%)$ y altos valores del sustrato abiótico (>50\%), los cuales estuvieron posiblemente asociados a varios de los hechos indicados. Uno de los sucesos de mayor impacto fue la mortandad masiva de las especies coralinas dominantes en ese momento, A. palmata y A. cervicornis, debido a la enfermedad de la banda blanca, la cual diezmó sus poblaciones en Colombia y demás regiones del Caribe (Díaz et al., 2000; Porter et al., 2001; Garzón-Ferreira y Díaz, 2003; Sutherland et al., 2004; Green et al., 2008).
In the PNNCRSB and specifically in the islands of San Bernardo, fishing on hydrobiological resources (Martínez-Viloria et al., 2011), tourist activity (Mendoza et al., 2011), use of biological material for the elaboration of crafts and souvenirs (Ordosgoitia and Zarza-González; 2011), and coral bleaching (Gómez-Campo et al., 2011) have been identified as the main threats.

Other local threats that can be added are the possible influence of sediments and freshwater from the Sinú River and hydrocarbons from the transit of tourist boats, and accidental spills due to activities associated with the Coveñas maritime terminal. The influence of freshwater is evidenced in the alkalinity concentrations both in the rainy season (107.5 to $116.6 \mathrm{mg} / \mathrm{L})$ and in the dry season (119.2 to $130.8 \mathrm{mg} / \mathrm{L}$ ) of 2013 in San Bernardo (Hyser, 2014). These values are below the ideal limits $(175-200 \mathrm{mg} / \mathrm{L})$ and even acceptable (124-225 mg/L) established for the culture of corals (Borneman, 2008), which limits calcification and reduces the rate of growth and skeletal density (Borneman, 2008; Hurtado, 2015).

In the case of hydrocarbons, the results of Betancourt-Portela et al. (2011), who are part of the monitoring carried out by Invemar's REDCAM, found concentrations below the international reference level of $10 \mu \mathrm{g} / \mathrm{L}$ in the PNNCRSB. Likewise, this document showed that since 2003 all samplings have registered a low level of contamination that does not represent a risk to aquatic organisms. There is also no evidence that the spills that have occurred in the Gulf of Morrosquillo have come to affect the coral formations of the San Bernardo archipelago (Carsucre, 2014).

In the San Bernardo archipelago, between 1989 and 1991 there was a low coral cover $(<20 \%)$ and high values of the abiotic substrate ( $>50 \%$ ). which were possibly associated with several of the events indicated. One of the events with the greatest impact was the mass death of the dominant coral species at that time, A. palmata and $A$. cervicornis, due to the white band disease, which decimated their populations in Colombia and other regions of the Caribbean (Díaz et al., 2000; Porter et al., 2001; Garzón-Ferreira and Díaz, 2003; Sutherland et al., 2004; Green et al., 2008).

Before this event, Erhardt and Meinel (1975) and Erhardt and Prahl (1985) mention the presence of large coral patches, as well as extensive hedges of the genus Acropora mainly on the reef crest and numerous colonies 
Previo a este suceso, Erhardt y Meinel (1975) y Erhardt y Prahl (1985) mencionan la presencia de grandes parches coralinos, así como de extensos setos del género Acropora principalmente en la cresta arrecifal y numerosas colonias de corales mixtos en sotavento. Asimismo, en Ceycen, observaron la dominancia de corales foliáceos en el borde del talud, los cuales fueron reemplazados por corales masivos a partir de los $12 \mathrm{~m}$ de profundidad. Posteriormente, en los resultados del monitoreo de 1989, Ramírez (1990) destaca el deterioro coralino, ya que el coral muerto (16,4\%), que pasó a formar parte del sustrato abiótico, fue superior a la cobertura coralina $(12,7 \%) \mathrm{y}$ fue el componente dominante del sustrato. Al parecer, los cambios posteriores entre este componente y las algas, se debieron en parte al cambio de investigadores en la campaña del 2013. Durante los seguimientos entre 1991 y 2010, los numerosos esqueletos de coral a pesar de estar colonizados por algas, fueron registrados como sustrato abiótico y desde el 2013 como algas. De este modo, es posible que la dominancia de este último componente se haya presentado realmente desde la década de 1990.

El incremento en la cobertura y dominancia de las algas, que persiste en la actualidad, ha sido documentado en otros arrecifes en Colombia y en el Caribe desde 1990, evidenciándose un cambio de fase, donde los arrecifes son dominados por algas y no por corales (Done, 1992; Hughes, 1994; Díaz et al., 2000; Garzón-Ferreira y Díaz, 2003; Díaz-Pulido et al., 2004; Rodríguez-Ramírez et al., 2010; Bastidas et al., 2014; Jackson et al., 2014). Los resultados de este monitoreo coinciden con esta condición, ya que históricamente, a excepción de 1999, la cobertura de las algas fue superior a la coralina. Este escenario evidencia el proceso de deterioro que han enfrentado los arrecifes del archipiélago de San Bernardo en las últimas décadas como consecuencia de factores de perturbación como enfermedades coralinas, blanqueamiento, cambio climático, turismo masivo, sobrepesca, contaminación, y desarrollo costero, entre otros, tal como ha ocurrido en otros arrecifes alrededor del mundo (Hughes, 1994; McClanahan y Mutinga, 1998; Buddemeier et al., 2004; Díaz-Pulido et al. 2004; Rodríguez-Ramírez et al., 2010; Alvarado et al., 2011).

Adicionalmente, la reducción en la cobertura coralina registrada en el 2005 estuvo posiblemente asociada al blanqueamiento masivo que se presentó ese mismo año, el cual fue uno de los eventos más severos y que produjo mortalidades coralinas en el Gran Caribe of mixed corals in the leeward. Likewise, in Ceycen, they observed the dominance of foliaceous corals at the edge of the slope, which was replaced by massive corals from $12 \mathrm{~m}$ depth. Later, in the monitoring results of 1989, Ramírez (1990) highlights the coral deterioration, since the dead coral (16.4\%), which became part of the abiotic substrate, was higher than the coral cover $(12.7 \%)$ and was the dominant component of the substrate. The later changes between this component and the algae were due in part to the change of researchers in the 2013 campaign. During the follow-ups between 1991 and 2010, the numerous coral skeletons, despite being colonized by algae, were recorded as an abiotic substrate and in 2013 as algae. Thus, the dominance of this last component may have been present since the 1990s.

The increase in the coverage and dominance of the algae, which persists today, has been documented in other reefs in Colombia and the Caribbean since 1990, evidencing a change of phase, where the reefs are dominated by algae and not by corals (Done, 1992; Hughes, 1994; Díaz et al., 2000; Garzón-Ferreira and Díaz, 2003; Díaz-Pulido et al., 2004; Rodríguez-Ramírez et al., 2010; Bastidas et al., 2014; Jackson et al., 2014). The results of this monitoring coincide with this condition, since historically, except for 1999, the cover of algae was higher than that of coralline. This scenario shows the deterioration process that the reefs of the San Bernardo archipelago have faced in recent decades as a consequence of disturbance factors such as coral diseases, bleaching, climate change, mass tourism, overfishing, pollution, and coastal development, among others occurring in other reefs around the world (Hughes, 1994; McClanahan and Mutinga, 1998; Buddemeier et al., 2004; Díaz-Pulido et al. 2004; Rodríguez-Ramírez et al., 2010; Alvarado et al., 2011).

Additionally, the reduction in coral cover recorded in 2005 was possibly associated with the massive bleaching that occurred that same year, which was one of the most severe events and produced coral mortalities in the Greater Caribbean (Wilkinson and Souter, 2008; Eakin et al., 2010). In the reef formations of the archipelago and surrounding areas, this event severely affected between 1 and $70 \%$ of the corals in the first $12 \mathrm{~m}$ of depth, with an associated mortality of $5 \%$ (Rodríguez-Ramírez et al., 2008; Gómez-Campo et al., 2011).

However, bleaching could be a determining factor in coral mortality, threatening the future of 
(Wilkinson y Souter, 2008; Eakin et al., 2010). En las formaciones arrecifales del archipiélago y áreas aledañas, este evento afectó severamente entre 1 y $70 \%$ de los corales en los primeros $12 \mathrm{~m}$ de profundidad, con una mortalidad asociada de $5 \%$ (Rodríguez-Ramírez et al., 2008; Gómez-Campo et al., 2011).

No obstante, el blanqueamiento podría ser un factor determinante en la mortandad coralina, amenazando el futuro de los arrecifes, considerando que estos eventos son cada vez más frecuentes (Hughes et al., 2017, 2018; Oliver et al., 2018). Adicionalmente, la respuesta adaptativa que pueden desarrollar los corales después de un blanqueamiento es demasiado lenta para mantenerse al día con la velocidad actual del cambio climático (Fabricius et al., 2007; Ainsworth et al., 2016).

Para el Gran Caribe, Jackson et al. (2014) documentaron una tendencia general a la reducción en la cobertura coralina, en promedio cercano a $20 \%$, entre la década de 1980 y 2011. Este mismo patrón fue observado en los arrecifes del Caribe colombiano (Díaz et al., 2000; Alvarado et al., 2011). Según la información del programa de monitoreo SIMAC, la cobertura coralina en cinco áreas arrecifales fue relativamente estable desde 1998 hasta 2004 (Rodríguez-Ramírez et al., 2010). Resultados posteriores, hasta el 2013, sugieren que las áreas de Santa Marta y las islas de San Bernardo permanecen estables, mientras que en las islas del Rosario, San Andrés y Urabá chocoano existe una tendencia al descenso (Bastidas et al., 2014). Similar a lo reportado por el SIMAC, en el presente monitoreo en el archipiélago de San Bernardo los corales presentaron una tendencia general a la estabilidad entre 1989 y 2010.

La riqueza de especies coralinas observada en este monitoreo (40 especies) es cercana a la documentada en el área de estudio de 49 y al de otras regiones como San Andrés y Urabá chocoano, ambas con 41 especies, Providencia con 49 especies, las islas del Rosario y Santa Marta, ambas con 53 especies (Prahl y Erhardt, 1985; Díaz et al., 2000). No obstante, esta información debe ser tomada con cautela, ya que la evaluación estuvo restringida a los primeros $12 \mathrm{~m}$, lo cual pudo excluir otros hábitats y formaciones coralinas.

Históricamente los corales con mayor cobertura y que más contribuyeron al ensamblaje coralino fueron Orbicella spp., Porites porites, Agaricia spp., P. astreoides y Siderastrea spp., por lo que pueden ser consideradas actualmente como los principales constructores de las formaciones coralinas estudiadas. Este esquema se ajusta en cierta forma a lo documentado por otros estudios (Alvarado reefs, considering that these events are increasingly frequent (Hughes et al., 2017, 2018; Oliver et al., 2018). Additionally, the adaptive response that corals can develop after bleaching is too slow to keep up with the current speed of climate change (Fabricius et al., 2007; Ainsworth et al., 2016).

For the Greater Caribbean, Jackson et al. (2014) documented a general trend towards a reduction in coral cover, on average close to $20 \%$, between the 1980 s and 2011. This same pattern was observed in the reefs of the Colombian Caribbean (Díaz et al., 2000; Alvarado et al., 2011). According to information from the SIMAC monitoring program, coral cover in five reef areas was relatively stable from 1998 to 2004 (Rodríguez-Ramírez et al., 2010). Subsequent results, until 2013, suggest that the areas of Santa Marta and the islands of San Bernardo remain stable, while in the Rosario Islands, San Andrés, and Urabá Chocoano there is a downward trend (Bastidas et al., 2014). Similar to what was reported by SIMAC, in the present monitoring in the San Bernardo archipelago, the corals showed a general trend towards stability between 1989 and 2010.

The richness of coral species observed in this monitoring (40 species) is close to that documented in the study area of 49 and that of other regions such as San Andrés and Urabá Chocoano, both with 41 species, Providencia with 49 species, the Rosario Islands and Santa Marta, both with 53 species (Prahl and Erhardt, 1985; Díaz et al., 2000). However, this information should be taken with caution, since the evaluation was restricted to the first $12 \mathrm{~m}$, which could exclude other habitats and coral formations.

Historically, the corals with the greatest coverage and that contributed the most to the coral assembly were Orbicella spp., Porites porites, Agaricia spp., P. astreoides and Siderastrea spp., which is why they can be considered today as the main constructors of the coral formations studied. This scheme is somewhat consistent with what has been documented by other studies (Alvarado et al., 1986; Cendales et al., 2002; Rodríguez-Ramírez et al., 2010; Alvarado et al., 2011) in the coral formations surrounding Barú and the Rosario Islands.

According to the records of Erhardt and Meinel (1975) and Erhardt and Prahl (1985), before the 1980s, although the Acropora genus was abundant, they also described the presence of numerous coralline species homogeneously distributed in the archipelago. However, 
et al., 1986; Cendales et al., 2002; Rodríguez-Ramírez et al., 2010; Alvarado et al., 2011) en las formaciones coralinas aledañas a Barú y a las islas del Rosario.

De acuerdo con los registros de Erhardt y Meinel (1975) y Erhardt y Prahl (1985), antes de la década de 1980, si bien el género Acropora fue abundante también describieron la presencia de numerosas especies coralinas distribuidas homogéneamente en el archipiélago. No obstante, $P$. porites fue más comúnmente observado en la laguna arrecifal entremezclado con Millepora spp. Las colonias de S. siderea y P. astreoides, cuyo diámetro era menor a $30 \mathrm{~cm}$ de diámetro, se encontraron principalmente como rodolitos en sustrato inestables. Adicionalmente, en algunas zonas protegidas del oleaje, A. tenuifolia fue dominante en parches a $6 \mathrm{~m}$ de profundidad. Por su parte, colonias masivas de Orbicella y Colpophyllia fueron abundantes a profundidades mayores a $12 \mathrm{~m}$.

Como consecuencia de la mortalidad de los acropóridos en los arrecifes del Caribe, incluyendo a Colombia, Bruckner (2002), Cendales et al. (2002) y Green et al. (2008) afirman que se presentó un cambio en la composición de la comunidad coralina, en el que especies pioneras con estrategias oportunistas como $A$. tenuifolia, $P$. astroides y especies de crecimiento lento con alta tasa de supervivencia como las del género Orbicella y Siderastrea están ocupando el lugar de los acropóridos. Estos cambios han traído como resultado la presencia de especies de coral capaces de vivir bajo condiciones de estrés. El aumento en la cobertura coralina en 2013 puede estar asociado al cambio de investigadores en este seguimiento. Sin embargo, podría haber una contribución por el crecimiento de estas especies con eficaces estrategias de vida, aunque no existen mediciones que demuestren el crecimiento de estas colonias durante este periodo.

A lo largo del monitoreo, la cobertura de acropóridos fue muy baja, menor a $1 \%$. Esto sugiere que en general, en el área evaluada, las poblaciones de este grupo han tenido una baja recuperación. Estudios realizados en diferentes años enfocados en este género coralino en el Caribe colombiano concluyeron que, a pesar de poseer una alta tasa de crecimiento, no hay evidencias de su real recuperación (Díaz et al., 2000; Garzón-Ferreira et al., 2004). Adicionalmente, aunque García-Urueña y GarzónMachado (2020), documentaron diferentes respuestas en su condición según la especie y su ubicación, el género Acropora en el archipiélago de San Bernardo no mostró signos de mejoría, especialmente A. cervicornis.
P. porites were more commonly observed in the reef lagoon intermixed with Millepora spp. The colonies of $S$. siderea and $P$. astreoides, whose diameter was less than $30 \mathrm{~cm}$ in diameter, were found mainly as rhodoliths in an unstable substrate. Additionally, in some areas protected from waves, A. tenuifolia was dominant in patches at $6 \mathrm{~m}$ depth. On the other hand, massive colonies of Orbicella and Colpophyllia were abundant at depths greater than $12 \mathrm{~m}$.

As a consequence of the mortality of acroporids on Caribbean reefs, including Colombia, Bruckner (2002), Cendales et al. (2002), and Green et al. (2008) state that there was a change in the composition of the coral community, in which pioneer species with opportunistic strategies such as A. tenuifolia, P. astroides, and slowgrowing species with a high survival rate such as those of the genus Orbicella and Siderastrea are taking the place of the acropóridos. These changes have resulted in the presence of coral species capable of living under stressful conditions. The increase in coral cover in 2013 may be associated with the change in researchers in this follow-up. However, there could be a contribution by the growth of these species with effective life strategies, although there are no measurements that demonstrate the growth of these colonies during this period.

Throughout the monitoring, the coverage of acroporids was very low, less than $1 \%$. This suggests that in general, in the evaluated area, the populations of this group have had a low recovery. Studies carried out in different years focused on this coral genus in the Colombian Caribbean, concluded that, despite having a high growth rate, there is no evidence of its real recovery (Díaz et al., 2000; Garzón-Ferreira et al., 2004). Additionally, although García-Urueña and Garzón-Machado (2020) documented different responses in their condition according to the species and its location, the genus Acropora in the San Bernardo archipelago did not show signs of improvement, especially A. cervicornis.

Considering the location of the stations within the archipelago, greater coverage of algae was evidenced, and generally less coral coverage, in the sectors that are closer to the continent such as Cabruna and Palma. In these sectors, there is a greater influence of disturbance factors of human origin, so that these coral formations probably face a continuous process of threats due to the activities that take place in the coastal zone and that lead to deterioration. Indicators such as a decrease in coral cover 
Considerando la ubicación de las estaciones dentro del archipiélago, se evidenció una mayor cobertura de algas y, por lo general, una menor cobertura de coral en los sectores que se encuentran más cerca del continente como Cabruna y Palma. En estos sectores existe una mayor influencia de factores de perturbación de origen humano, por lo que estas formaciones coralinas probablemente enfrentan un proceso continuo de amenazas por las actividades que se desarrollan en la zona costera y que las conducen al deterioro. Indicadores como un descenso de la cobertura coralina a lo largo del monitoreo fue registrado en varias de las estaciones de estos sectores (C-15, C-18 y C-19). La continua exposición de estas formaciones coralinas a los tensores causantes de deterioro coralino podría limitar su capacidad de recuperación, amenazando su persistencia (Hughes, 1994; Buddemeier et al., 2004; Hughes et al., 2010; Burke et al., 2011). Este deterioro afecta la estructura y la dinámica del ecosistema y representa una amenaza permanente para el mantenimiento de los bienes y servicios que se derivan de él.

Por el contrario, hay formaciones coralinas que mostraron mejores condiciones de estado como C-6, C-11 y C-14, ubicadas al N y NO de las islas Múcura, Tintipán y Mangle, respectivamente. En varias de estas estaciones se observó una mayor presencia de corales juveniles y un incremento en la cobertura de coral en el tiempo. Estas estaciones podrían estar entre los lugares del archipiélago que se encuentran mejor conservados y donde existe una buena probabilidad de incrementarse la presencia de corales juveniles. Por lo tanto, se debe tener especial atención en su gestión y protección, ya que podrían ser clave en el mantenimiento de las comunidades coralinas del área.

Los arrecifes de coral tienen un ámbito estrecho de tolerancia térmica, entre 18 y $36^{\circ} \mathrm{C}$ con un intervalo óptimo entre 25 y $29^{\circ} \mathrm{C}$ (Stoddar, 1969; Hubbard, 1997; Raymundo et al., 2008), por lo que este ecosistema es extremadamente susceptible al estrés térmico (Harvell et al., 2007). En 2015 las formaciones coralinas enfrentaron un estrés causado por las altas temperaturas acumuladas de meses atrás que estuvo reflejado, en parte, en la presencia del blanqueamiento en 18 de las 19 estaciones evaluadas y el registro de algunas enfermedades. Sin embargo, su impacto fue bajo, ya que las colonias fueron afectadas sólo de forma parcial. No obstante, a finales del 2015 y en el 2016 se registró un evento masivo de blanqueamiento global, asociado a altas temperaturas, que afectó severamente a los arrecifes del Pacífico Occidental y en Colombia fue considerado como throughout the monitoring were recorded in several of the stations in these sectors (C-15, C-18, and C-19). The continuous exposure of these coral formations to tensors that cause coral deterioration could limit their capacity for recovery, threatening their persistence (Hughes, 1994; Buddemeier et al. 2004; Hughes et al., 2010; Burke et al., 2011). This deterioration affects the structure and dynamics of the ecosystem and represents a permanent threat to the maintenance of the goods and services derived from it.

On the contrary, there are coral formations that showed better state conditions such as C-6, C-11, and C-14, located to the $\mathrm{N}$ and $\mathrm{NW}$ of the Múcura, Tintipán, and Mangle islands, respectively. In several of these stations, a greater presence of juvenile corals and an increase in coral cover were observed over time. These stations could be among the best-conserved places in the archipelago and where there is a good probability of increasing the presence of juvenile corals. Therefore, special attention should be paid to their management and protection, since they could be key in the maintenance of the coral communities in the area.

Coral reefs have a narrow range of thermal tolerance, between 18 and $36{ }^{\circ} \mathrm{C}$ with an optimal range between 25 and $29^{\circ} \mathrm{C}$ (Stoddar, 1969; Hubbard, 1997; Raymundo et al., 2008), so this ecosystem is extremely susceptible to heat stress (Harvell et al., 2007). In 2015, the coral formations faced stress caused by the high temperatures accumulated months ago, which was reflected, in part, in the presence of bleaching in 18 of the 19 stations evaluated and the registration of some diseases. However, its impact was low, as the colonies were only partially affected. However, at the end of 2015 and in 2016 a massive global bleaching event was registered, associated with high temperatures, which severely affected the reefs of the Western Pacific and in Colombia, it was considered a low-level event (Hughes et al., 2017, 2018; Oliver et al., 2018).

From the perspective of resilience, the protection that national parks can provide is only one part of the solution for the conservation of ecosystems, since this must be accompanied by other mechanisms such as strong management measures (Hughes et al., 2010) and ecological restoration (Pizarro et al., 2014). Likewise, management actions on local impacts such as overfishing, tourist activity, degradation of water quality, pollution, deforestation, among others, are essential to stop the deterioration and begin to protect coral reefs to adapt to 
un evento de nivel bajo (Hughes et al., 2017, 2018; Oliver et al., 2018).

Desde la perspectiva de la resiliencia, la protección que pueden proveer los parques nacionales es sólo una parte de la solución para la conservación de los ecosistemas, ya que esta debe ir acompañada de otros mecanismos como fuertes medidas de manejo (Hughes et al., 2010) y restauración ecológica (Pizarro et al., 2014). Igualmente, son esenciales las acciones de gestión sobre los impactos locales como la sobrepesca, la actividad turística, la degradación de la calidad del agua, la contaminación, la deforestación, entre otros, para detener el deterioro y comenzar a proteger los arrecifes coralinos para que puedan adaptarse a los cambios ambientales y así aumentar su recuperación frente al cambio climático (Wooldridge et al., 2005; Hughes et al., 2007, 2010; Knowlton y Jackson, 2008). Esto va de la mano con la continuidad de los programas de monitoreo para generar información sobre el estado, tendencias y cambios de los ecosistemas marinos.

\section{CONCLUSIONES}

Esta evaluación de largo plazo evidenció una dinámica opuesta y una gran variación en la cobertura del sustrato abiótico y de las algas, siendo este último grupo el componente más abundante del fondo marino desde el 2013 y que corresponde al panorama general observado en los arrecifes coralinos a nivel mundial. El ensamblaje coralino presentó una tendencia estable entre 1989 y 2010. Sin embargo, la disimilitud temporal registrada en el 2013 y 2015 con los demás años fue a causa de las mayores coberturas de los corales Orbicella spp., P. porites, Agaricia spp., Siderastrea spp. y $M$. complanata, cuyas estrategias de vida, con la excepción de $M$. complanata, les ha permitido llegar a ser los principales constructores de las formaciones coralinas del archipiélago de San Bernardo. La dinámica temporal de los principales componentes del sustrato es consecuencia del efecto de las perturbaciones naturales y antropogénicas a las que han sido sometidos estos arrecifes en las últimas décadas. No obstante, a nivel espacial se puede apreciar una gran variabilidad de coberturas coralinas donde se destacan arrecifes ubicados en las islas Múcura, Tintipán y Mangle que presentaron mejores condiciones de estado. Adicionalmente, la mayor frecuencia de observación de blanqueamiento en el 2015, que coincidió con el registro environmental changes and thus increase their recovery from climate change (Wooldridge et al., 2005; Hughes et al., 2007, 2010; Knowlton and Jackson, 2008). This goes hand in hand with the continuity of monitoring programs to generate information on the state, trends, and changes of marine ecosystems.

\section{CONCLUSIONS}

This long-term evaluation showed an opposite dynamic and a great variation in the cover of the abiotic substrate and algae, the latter group being the most abundant component of the seabed since 2013 and corresponding to the general panorama observed in the coral reefs at the world level. The coral assemblage presented a stable trend between 1989 and 2010. However, the temporal dissimilarity registered in 2013 and 2015 with the other years was due to the greater coverage of the corals Orbicella spp., P. porites, Agaricia spp., Siderastrea spp., and $M$. complanata, whose life strategies, except for $M$. complanata, have allowed them to become the main builders of the coral formations of the San Bernardo archipelago. The temporal dynamics of the main components of the substrate are a consequence of the effect of natural and anthropogenic disturbances to which these reefs have been subjected in recent decades. However, at a spatial level, great variability of coralline covers can be appreciated, where reefs located on the Múcura, Tintipán, and Mangle islands stand out, presenting better state conditions. Additionally, the higher frequency of observation of bleaching in 2015, which coincided with the record of high temperatures, shows that SST is an important disturbing factor that is currently affecting coral formations.

\section{ACKNOWLEDGEMENTS}

This study was conducted in the framework of the Monitoring of Natural Marine Ecosystem of the Environmental Management Plan Program of the Covenas terminal by Ecopetrol S.A., which we greatly appreciated their support and funding. The authors thank the company Hyser Ltda. for their support in the management and implementation of this research. To the National Natural Park Corales of the Rosario and San Bernardo for his accompaniment. Finally, to Shirly Bello-Escobar for the development of cartography and Milena Marrugo, Alejandro Henao, David Acevedo, and Diego Quiroga for their support in field activities. 
de altas temperaturas, pone en evidencia que la TSM es un factor de perturbación importante que actualmente están afectando a las formaciones coralinas.

\section{AGRADECIMIENTOS}

Este estudio se desarrolló en el marco del programa de Seguimiento y Monitoreo de Ecosistemas Naturales Marinos del Plan de Manejo Ambiental del Terminal de Coveñas por Ecopetrol S.A., al cual se agradece enormemente por su apoyo y financiación. Los autores también agradecen a la empresa Hyser Ltda. por todo su apoyo en la gestión y realización de esta investigación. Asimismo, al Parque Nacional Natural Corales del Rosario y San Bernardo por su acompañamiento. Por último, a Shirly Bello-Escobar por la elaboración de la cartografía y a Milena Marrugo, Alejandro Henao, David Acevedo y Diego Quiroga por el apoyo en las actividades de campo.

\section{BIBLIOGRAFÍA/LITERATURE CITED}

Ainsworth, T., S. Heron, J.C. Ortiz, P. Mumby, A. Grech, D. Ogawa, C.M. Eakin, and W. Leggat. 2016. Climate change disables coral bleaching protection on the Great Barrier Reef. Science, 352(6283): 338-342.

Anderson, M. 2001. A new method for non-parametric multivariate analysis of variance. Austral Ecol., 26: 32-46.

Andrade-Amaya, C.A. 2001. Las corrientes superficiales en la cuenca de Colombia observadas con boyas de deriva. Rev. Acad. Colomb. Cienc. Ex. Fis. Nat., 25 (96): 321-335.

Alvarado, E.M., F. Duque, L. Flórez y R. Ramírez. 1986. Evaluación cualitativa de los arrecifes coralinos de las islas del Rosario (Cartagena, Colombia). Bol. Ecotrópica, 15: 1-30.

Alvarado, E., V. Pizarro y A. Sarmiento-Segura. 2011. Formaciones arrecifales. 109-123. En: Zarza-González, E. (Ed). El entorno ambiental del Parque Nacional Natural Corales del Rosario y San Bernardo. Ministerio de Medio Ambiente y Desarrollo Sostenible, Parques Nacionales Naturales de Colombia, Banco Mundial - GEF, Patrimonio Natural - Fondo para Biodiversidad y las Áreas Protegidas, Cartagena de Indias, Colombia. 416 p.

Bastidas, M., N. Rangel, D. Morales, C. Ricaurte, D.I. Gomez-López, R. Navas-Camacho, S.M. Navarrete, D. Alonso, L.J. Vivas-Aguas, P.S. Obando, J.A. Rodríguez-Rodríguez, L.V. Licero-Villanueva y L. Perdomo. 2014. Estado del ambiente y los ecosistemas marinos y costeros. 23-82. En: Informe del estado de los ambientes y recursos marinos y costeros en Colombia: año 2013. Ser. Publ. Per. Invemar, (3), Santa Marta. 192 p.

Bernhard, R. 2007. Extreme climatic events and coral reefs: How much short-term threat from global change? 315-341. In: Aronson R.B. (Eds). Geological approaches to coral reef ecology. Springer, New York. 439 p. https://doi.org/10.1007/978-0-387-33537-7_10

Betancourt-Portela, J.M., L.F. Espinosa, S. Narváez, J. Vivas-Aguas y J.P. Parra. 2011. 67-77. En: Zarza-González, E. (Ed). El entorno ambiental del Parque Nacional Natural Corales del Rosario y de San Bernardo. Ministerio de Medio Ambiente y Desarrollo Sostenible, Parques Nacionales Naturales de Colombia, Banco Mundial - GEF, Patrimonio Natural - Fondo para Biodiversidad y las Áreas Protegidas, Cartagena de Indias, Colombia. $416 \mathrm{p}$.

Birkeland, C. 1997. Life and death of corals reefs. Chapman \& Hall, Nueva York. 536 p.

Borneman, E. 2008. Introduction to the husbandry of corals in aquariums: A review. 3-14. In: Leewis, J. and M. Janse (Eds.). Advances in coral husbandry in public aquariums. Public Aquarium Husbandry Series, 2. Burgers’ Zoo, Arnhem. 444 p.

Bruckner, A. 2002. Proceedings of the Caribbean Acropora Workshop: Potential application of the U.S. endangered species act as a conservation strategy. NOAA Technical Memorandum NMFS-OPR-24. Silver Spring, USA. 184 p. 
Buddemeier, R.W., J.A. Kleypas, and R.B. Aronson. 2004. Coral reefs and global climate change: Potential contributions of climate change to stresses on coral reef ecosystems. Pew Center on Global Climate Change, Arlington. 44 p.

Burke, L., K. Reytar, M. Spalding, and A. Perry. 2011. Reef at risk: revisited. World Resources Institute, Washington. 114 p.

Carsucre, 2014. Informe de seguimiento en el sector de la parte trasera de la ciénaga de La Caimanera, municipio de Coveñas y en sector de las playas del Francés-Alegría, vía Guacamayas, municipio de Santiago de Tolú, por derrames de hidrocarburos. Informe final, Sincelejo. 7 p.

Cendales, M., S. Zea y J. Díaz. 2002. Geomorfología y unidades ecológicas del complejo de arrecifes de las Islas del Rosario e isla Barú (mar Caribe, Colombia). Rev. Acad. Colomb. Cienc., 26(101): 497-510.

Clarke, K.R. 1993. Non-parametric multivariate analyses of changes in community structure. Aus. J. Ecol., 18: 117-143.

Clarke, K.R. and R.N. Gorley. 2015. PRIMER v7: User manual/tutorial. PRIMER-E: Plymouth. Devon, UK. 296 p.

Díaz, J.M., L.M. Barrios, M.H. Cendales, J. Garzón-Ferreira, J. Geister, M. López-Victoria, G.H. Ospina, F. Parra-Velandia, J. Pinzón, B. Vargas-Ángel, F.A. Zapata y S. Zea. 2000. Áreas coralinas de Colombia. Invemar. Ser. Publ. Esp. Invemar, (5), Santa Marta. 179 p.

Díaz-Pulido, G., J. Sánchez, S. Zea, J. Díaz y J. Garzón-Ferreira. 2004. Esquemas de distribución espacial en la comunidad bentónica de arrecifes coralinos continentales y oceánicos del Caribe colombiano. Rev. Acad. Colomb. Cienc., 24(108): 337-347.

Done, T.J. 1992. Phase shifts in coral reef communities and their ecological significance. Hydrobiologia, 247: 121-132.

Duque, F. y H. Gómez. 1983. El archipiélago de San Bernardo y su fauna íctica. Tesis Biol. Mar., Univ. Jorge Tadeo Lozano, Bogotá. 90 p.

Duque-Rico, M.A. y L. Torres-Gómez. 2011. Aspectos socioeconómicos de las principales comunidades del archipiélago de San Bernardo, sector Barú y archipiélago de Nuestra Señora del Rosario. 26-37. En: Zarza-González, E. (Ed). El entorno ambiental del Parque Nacional Natural Corales del Rosario y de San Bernardo. Ministerio de Medio Ambiente y Desarrollo Sostenible, Parques Nacionales Naturales de Colombia, Banco Mundial GEF, Patrimonio Natural - Fondo para Biodiversidad y las Áreas Protegidas, Cartagena de Indias, Colombia. 416 p.

Eakin, C.M., J.A. Morgan, S.F. Heron, T.B. Smith, G. Liu, L. Álvarez-Filip, B. Baca, et al. 2010. Caribbean corals in crisis: record thermal stress, bleaching, and mortality in 2005. PLoS ONE, 5(11): e13969. https://doi.org/10.1371/journal.pone.0013969

Erhardt, H. and W. Meinel. 1975. Die scleractinen Korallen der Insel Ceycén, Islas San Bernardo, vor der kolumbianischen Atlanfikküste. Philippia, 2: 236-247.

Fabricius, K., O. Hoegh-Guldberg, J. Johnson, L. McCook, and J. Lough. 2007. Vulnerability of coral reefs of the Great Barrier Reef to climate change. 515-554. In: Johnson, J. y Marshall P.A. (Eds). Climate change and the Great Barrier Reef. Chapter 17. Great Barrier Marine Park Authority and Australian Greenhouse Office, Australia. 801 p.

García-Urueña, R. and M.A. Garzón-Machado. 2020. Current status of Acropora palmata and Acropora cervicornis in the Colombian Caribbean: demography, coral cover and condition assessment. Hydrobiologia, 847: 2141-2153. https://doi.org/10.1007/s10750-020-04238-6

Garzón-Ferreira, J., M.C. Reyes-Nivia y A. Rodríguez-Ramírez. 2002. Manual de métodos del SIMAC - Sistema Nacional de Monitoreo de Arrecifes Coralinos en Colombia. Informe final, Invemar, Santa Marta. 61 p.

Garzón-Ferreira, J. and J.M. Díaz. 2003. The Caribbean coral reefs of Colombia. 275-301. En: Cortés, J. (Ed.). Latin American Coral Reefs. Elsevier Science B.V., Ámsterdam. 508 p.

Garzón-Ferreira, J., M. Moreno-Bonilla y J.M. Valderrama. 2004. Condición actual de las formaciones coralinas de Acropora palmata y A. cervicornis en el Parque Nacional Natural Tayrona (Colombia). Bol. Invest. Mar. Cost., 33: 117-136.

Gómez-Campo, K., T. López-Londoño, D.L. Gil-Agudelo, R. Navas-Camacho, J.A. Rojas y J. Garzón-Ferreira. 2011. Blanqueamiento coralino, amenaza para el futuro de los arrecifes de coral de los archipiélagos Nuestra Señora del Rosario y San Bernardo. 319-328. En: Zarza-González, E. (Ed). El entorno ambiental del Parque Nacional Natural Corales del Rosario y San Bernardo. Parques Nacionales Naturales de Colombia, Cartagena de Indias, Colombia. $416 \mathrm{p}$.

Green, D., P. Edmunds, and R. Carpenter. 2008. Increasing relative abundance of Porites astreoides on Caribbean reefs mediated by an overall decline in coral cover. Mar. Ecol. Prog. Ser., 359: 1-10.

Gutiérrez-Moreno, C., M. Marrugo, P. Lozano-Rivera, P. C. Sierra y C. Andrade. 2011. Clima marino. 39-47. En: Zarza-González, E. (Ed). El entorno ambiental del Parque Nacional Natural Corales del Rosario y de San Bernardo. Ministerio de Medio Ambiente y Desarrollo Sostenible, Parques 
Nacionales Naturales de Colombia, Banco Mundial - GEF, Patrimonio Natural - Fondo para Biodiversidad y las Áreas Protegidas, Cartagena de Indias, Colombia. $416 \mathrm{p}$.

Harvell, D., E. Jordán-Dahlgren, S. Merkel, E. Rosenberg, L. Raymundo, G. Smith, E. Weil, and B. Willis. 2007. Coral diseases environmental drivers and the balance and balance between coral and microbial associates. Oceanography, 20(1): 172-195.

Hubbard, D. 1997. Reefs as dynamic systems. 43-67. In: Birkeland, C. (Ed). Life and death of coral reefs. Chapman y Hall, Nueva York, USA. 536 p.

Hughes, T.P. 1994. Catastrophes, phase-shifts, and large-scale degradation of a Caribbean coral-reef. Science, 265: 1547-1551.

Hughes, T.P., M.J. Rodríguez, D.R. Bellwood, D. Ceccarelli, O. Hoegh-Guldberg, and L. McCook. 2007. Phase shifts, herbivory, and the resilience of coral reefs to climate change. Curr. Biol., 17: 360-365.

Hughes, T.P., N. Graham, J. Jackson, P. Mumby, and P. Steneck. 2010. Rising to the challenge of sustaining coral reef resilience. Trends Ecol. Evol., 11: 633-642.

Hughes, T.P., J.T. Kerry, M. Álvarez-Noriega, J.G. Álvarez-Romero, K.D. Anderson, A.H. Baird, R.C. Babcock, et al. 2017. Global warming and recurrent mass bleaching of corals. Nature, 543: 373-377. https://doi.org/10.1038/nature21707

Hughes, T.P., K.D. Anderson, S.R. Connolly, S.F. Heron, J.T. Kerry, J.M. Lough, A.H. Baird, J.K. Baum, M.L. Berumen, T.C. Bridge, D.C. Claar, C.M. Eakin, J.P. Gilmour, N.A.J. Graham, H. Harrison, J.P.A. Hobbs, A.S. Hoey, M. Hoogenboom, R.J. Lowe, M.T. McCulloch, J.M. Pandolfi, M. Pratchett, V. Schoepf, G. Torda, and S.K. Wilson. 2018. Spatial and temporal patterns of mass bleaching of corals in the Anthropocene. Science, 359: 80-83. https://doi.org/10.1126/science.aan8048

Hurtado, N. 2015. Understanding coral growth: in situ and ex situ experiments with Caribbean species for restoration purposes. Tesis Mg. Cienc. Mar., Univ. Jorge Tadeo Lozano, Santa Marta. 122 p.

HYSER. 2014. Caracterización de los ecosistemas marinos en el Golfo de Morrosquillo. Monitoreo año 2013. Informe final, Coveñas. 310 p.

Invemar. 2005. Monitoreo de corales en el golfo de Morrosquillo. Informe final, Ecopetrol, Cúcuta.

Jackson, J.B.C., M.K. Donovan, K.L. Cramer, and V.V. Lam. 2014. Status and trends of Caribbean coral reefs: 1970-2012. Global Coral Reef Monitoring Network, IUCN, Gland, Switzerland. 254 p.

Knowlton, N. and J.B.C. Jackson. 2008. Shifting baselines, local impacts, and global change on coral reefs. PLoS Biol., 6: 215-220.

Liu, G., C.M. Eakin, M. Chen, A. Kumar, J.L. de la Cour, S.F. Heron, E.F. Geiger, W.J. Skirving, K.V. Tirak, and A.E. Strong. 2018. Predicting heat stress to inform reef management: NOAA Coral Reef Watch's 4-month coral bleaching outlook. Front. Mar. Sci., 5(57). https://doi.org/10.3389/ fmars.2018.00057.

López-Victoria, M. y J.M. Díaz. 2000. Morfología y estructura de las formaciones coralinas del archipiélago de San Bernardo, Caribe colombiano. Rev. Acad. Colomb. Cienc., 24(91): 219-230.

Martínez-Viloria, H.M., L.A. Martínez-Whisgman, A. Vargas-Pineda y J.C. Narváez-Barandica. 2011. Efectos de la pesca sobre los recursos hidrobiológicos del Parque Nacional Natural Corales del Rosario y de San Bernardo. 273-289. En: Zarza-González, E. (Ed). El entorno ambiental del Parque Nacional Natural Corales del Rosario y de San Bernardo. Ministerio de Medio Ambiente y Desarrollo Sostenible, Parques Nacionales Naturales de Colombia, Banco Mundial - GEF, Patrimonio Natural - Fondo para Biodiversidad y las Áreas Protegidas, Cartagena de Indias, Colombia. 416 p.

McClanahan, T. and N. Mutinga. 1998. An ecological shift in a remote coral atoll of Belize over 25 years. Environ. Conserv., 25(2): 122-130.

Mendoza, J., L.A. Castro, P. Herrón, C. Montaño y F. Castro. 2011. Características de la actividad turística. 305-318. En: Zarza-González, E. (Ed). El entorno ambiental del Parque Nacional Natural Corales del Rosario y de San Bernardo. Ministerio de Medio Ambiente y Desarrollo Sostenible, Parques Nacionales Naturales de Colombia, Banco Mundial - GEF, Patrimonio Natural - Fondo para Biodiversidad y las Áreas Protegidas, Cartagena de Indias, Colombia. 416 p.

Navas-Camacho, R., J. Vega-Sequeda, K. Gómez-Campo, T. López-Londoño, D. Gil-Agudelo, L. Muñoz-Escobar, J. Rojas y D.L. Duque. 2011. El "Sistema Nacional de Monitoreo de Arrecifes Coralinos en Colombia-SIMAC" en el Parque Nacional Natural Corales del Rosario y San Bernardo: 1998-2009. 330-350. En: Zarza-González, E. (Ed). El entorno ambiental del Parque Nacional Natural Corales del Rosario y San Bernardo. Ministerio de Medio Ambiente y Desarrollo Sostenible, Parques Nacionales Naturales de Colombia, Banco Mundial - GEF, Patrimonio Natural - Fondo para Biodiversidad y las Áreas Protegidas, Cartagena de Indias, Colombia. 416 p. 
NOAA Coral Reef Watch. 2020. Caribbean 5 km regional virtual station time series data. Colombia Atlantic (version 3.1). https://coralreefwatch.noaa. gov/product/vs/data/colombia_atlantic.txt. 06/07/2020.

Oliver, J.K., R. Berkelmans, and C.M. Eakin. 2018. Coral bleaching in space and time. 27-49. In: van Oppen, M. and J. Lough (Eds). Coral Bleaching. Patterns, processes, causes and consequences. Volume 233. Springer, Switzerland. https://doi.org/10.1007/978-3-319-75393-5_3

Ordosgoitia, Y. y E. Zarza-González. 2011. Características de la comercialización de material biológico marino para la elaboración de artesanías y venta de recordatorios. 290-304. En: Zarza-González, E. (Ed). El entorno ambiental del Parque Nacional Natural Corales del Rosario y de San Bernardo. Ministerio de Medio Ambiente y Desarrollo Sostenible, Parques Nacionales Naturales de Colombia, Banco Mundial - GEF, Patrimonio Natural Fondo para Biodiversidad y las Áreas Protegidas, Cartagena de Indias, Colombia. 416 p.

Patiño, F y F. Flores. 1993. Estudio ecológico del Golfo de Morrosquillo. Universidad Nacional de Colombia y Fondo FEN-Colombia, Bogotá. 295 p.

Pizarro, V., V. Carrillo y A. García-Rueda. 2014. Revisión y estado del arte de la restauración ecológica de arrecifes coralinos. Biota Colomb., 15(Supl. 2): $132-149$.

Prahl, H.V. y H. Erhardt. 1985. Colombia, corales y arrecifes Coralinos. Fondo FEN-Colombia, Bogotá. 295 p.

Porter, J., P. Dustan, W. Jaap, K. Patterson, V. Kosmynin, O. Meier, M. Patterson, and M. Parsons. 2001. Patterns of spread of coral disease in the Florida Keys. Hydrobiologia, 460: 1-24.

Pujos, M., J. L. Pagliardini, R. Steer, G. Vemette y O. Weber. 1986. Influencia de la contra-corriente norte colombiana para la circulación de las aguas en la plataforma continental su acción sobre la dispersión de los efluentes en suspensión del río Magdalena. Bol. Cient. CIOH, 6: 3-15.

Ramírez, A. 1990. Monitoreo de los ecosistemas coralinos del golfo de Morrosquillo y el archipiélago de San Bernardo. Fase II. Informe final, EcopetrolDCC, Cúcuta.

Ramírez, A. 1992. Los ecosistemas coralinos del archipiélago de San Bernardo. Golfo de Morrosquillo, mar Caribe. Informe final, Ecopetrol-DCC, Cúcuta.

Ramírez, A. 2000. Monitoreo de Corales en el golfo de Morrosquillo. Informe final, contrato Ecopetrol DCC-4AMT-47200-99-0007, Cúcuta.

Ramírez, A. 2010. Monitoreo de Corales en el golfo de Morrosquillo. Informe final, contrato Ecopetrol N. ${ }^{\circ} 4027656$, Bogotá. 119 p.

Raymundo, L., C. Couch, and C. Harvell. 2008. Coral disease handbook: guidelines for assessment monitoring and management. Coral Reef Targeted Research and Capacity Building for Management Program, Melbourne, Australia. 121 p.

Rodríguez-Ramírez, A., C. Bastidas, S. Rodríguez, Z. Leão, R. Kikuchi, M. Oliviera, D. Gil, J. Garzón-Ferreira, M.C. Reyes-Nivia, R. Navas-Camacho, N. Santodomingo, G. Díaz-Pulido, D. Venera-Pontón, L. Flórez-Leiva, A. Rangel-Campo, C. Orozco, J. C. Márquez, S. Zea, M. López-Victoria, J.A. Sánchez, and M.C. Hurtado. 2008. The effects of coral bleaching in Southern Tropical America: Brazil, Colombia, and Venezuela. 105-114. In: Wilkinson, C. and D. Souter (Eds). Status of Caribbean Coral Reefs after Bleaching and Hurricanes in 2005. Global Coral Reef Monitoring Network and Reef and Rainforest Research Centre, Townsville, Australia. 148 p.

Rodríguez-Ramírez, A., M.C. Reyes-Nivia, S. Zea, R. Navas-Camacho, J. Garzón-Ferreira, S. Bejarano, P. Herrón, and C. Orozco. 2010. Recent dynamics and condition of coral reefs in the Colombian Caribbean. Rev. Biol. Trop., 58(Suppl. 1): 107-131.

Stoddart, D. 1969. Ecology and morphology of recent coral reefs. Biol. Rev. Cambridge Phil. Soc., 44: 433-498.

Sutherland, K., J. Porter, and C. Torres. 2004. Diseases and immunity in Caribbean and Indo-Pacific zooxanthellate corals. Mar. Ecol. Prog. Ser., 266: 273-302.

Vega-Sequeda, J., S. Zea y G. Bernal. 2017. Efectos de eventos oceánicos extremos en formaciones coralinas de islas del Rosario, Caribe colombiano. Cicimar Oceánides, 32(1): 25-38.

Werding, B. y H. Sánchez. 1979. Informe faunístico y florístico de las islas del Rosario en la costa norte de Colombia. Situación general y estructuras arrecifales. An. Inst. Invest. Mar. Punta Betín, 11: 7-20.

Wilkinson, C. and D. Souter. 2008. Status of Caribbean coral reefs after bleaching and hurricanes in 2005. Global Coral Reef Monitoring Network y Reef and Rainforest Research Centre, Townsville. 148 p.

Wooldridge, S., T. Done, R. Berkelmans, R. Jones, and P. Marshall. 2005. Precursors for resilience in coral communities in a warming climate: a belief network approach. Mar. Ecol. Prog. Ser., 295: 157-169. https://doi.org/10.3354/meps295157 
Zarza-González, E. 2011. El Parque Nacional Natural Corales del Rosario y San Bernardo. 8-11. En: Zarza-González, E. (Ed). El entorno ambiental del Parque Nacional Natural Corales del Rosario y de San Bernardo. Ministerio de Medio Ambiente y Desarrollo Sostenible, Parques Nacionales Naturales de Colombia, Banco Mundial - GEF, Patrimonio Natural - Fondo para Biodiversidad y las Áreas Protegidas, Cartagena de Indias, Colombia. 416 p.

Zea, S. 1991. Establecimiento de estaciones base para monitorear el efecto de condiciones naturales y de origen humano en comunidades coralinas de Santa Marta y Parque Nacional Natural Tayrona. Informe final, Invemar, Santa Marta. 62 p.

RECIBIDO/RECEIVED: 09/08/2020

ACEPTADO/ACCEPTED: 01/11/2020 